\title{
Mec1, INO80, and the PAF1 complex cooperate to limit transcription replication conflicts through RNAPII removal during replication stress
}

\author{
Jérôme Poli, ${ }^{1,6}$ Christian-Benedikt Gerhold, ${ }^{1,6}$ Alessandro Tosi, ${ }^{2}$ Nicole Hustedt, ${ }^{1,5}$ \\ Andrew Seeber, ${ }^{1}$ Ragna Sack, ${ }^{1}$ Franz Herzog, ${ }^{2}$ Philippe Pasero, ${ }^{3}$ Kenji Shimada, ${ }^{1}$ \\ Karl-Peter Hopfner, ${ }^{2}$ and Susan M. Gasser ${ }^{1,4}$ \\ ${ }^{1}$ Friedrich Miescher Institute for Biomedical Research, CH-4058 Basel, Switzerland; ${ }^{2}$ Gene Center, Ludwig Maximilian University \\ of Munich, 81377 Munich, Germany; ${ }^{3}$ UPR 1142, Institut de Génétique Humaine, Centre National de la Recherche Scientifique, \\ 30396 Montpellier, France; ${ }^{4}$ Faculty of Natural Sciences, University of Basel, CH-4056 Basel, Switzerland
}

Little is known about how cells ensure DNA replication in the face of RNA polymerase II (RNAPII)-mediated transcription, especially under conditions of replicative stress. Here we present genetic and proteomic analyses from budding yeast that uncover links between the DNA replication checkpoint sensor Mec1-Ddc2 (ATR-ATRIP), the chromatin remodeling complex INO80C (INO80 complex), and the transcription complex PAF1C (PAF1 complex). We found that a subset of chromatin-bound RNAPII is degraded in a manner dependent on Mec1, INO80, and PAF1 complexes in cells exposed to hydroxyurea (HU). On HU, Mec1 triggers the efficient removal of PAF1C and RNAPII from transcribed genes near early firing origins. Failure to evict RNAPII correlates inversely with recovery from replication stress: paf1 $1 \Delta$ cells, like ino80 and mec1 mutants, fail to restart forks efficiently after stalling. Our data reveal unexpected synergies between INO80C, Mec1, and PAF1C in the maintenance of genome integrity and suggest a mechanism of RNAPII degradation that reduces transcription-replication fork collision.

[Keywords: transcription-replication interference; RNAPII; INO80; replication stress; checkpoint; genome instability] Supplemental material is available for this article.

Received October 21, 2015; revised version accepted December 21, 2015.

Genomes are particularly at risk as cells passage through $S$ phase. Perturbation of DNA replication leads to gross chromosomal rearrangements and translocations, which arise from replication fork collapse and double-strand breaks (DSBs) (Hustedt et al. 2013; Zeman and Cimprich 2014). The DNA replication checkpoint (DRC) limits genome instability by orchestrating multiple cellular responses that promote the maintenance and recovery of stalled forks (Branzei and Foiani 2010). Indeed, the sensor kinase Mec1-Ddc2 (ATR-ATRIP) recognizes ssDNA and its ligand, RPA, which accumulate at stalled replication forks, activating a kinase cascade known as the DRC (Tourriere and Pasero 2007).

Recent phosphoproteomic analyses have shown that many factors involved in chromatin remodeling and transcription, besides DNA replication and repair factors, are targets of checkpoint kinases (Bastos de Oliveira et al.

\footnotetext{
${ }^{5}$ Present address: The Lunenfeld-Tanenbaum Research Institute, Mount Sinai Hospital, Toronto, Ontario M5G 1X5, Canada

${ }^{6}$ These authors contributed equally to this work.

Corresponding author: susan.gasser@fmi.ch

Article published online ahead of print. Article and publication date are online at http://www.genesdev.org/cgi/doi/10.1101/gad.273813.115.
}

2015; Hustedt et al. 2015). Among these is the INO80 complex (INO80C), a 15-subunit nucleosome remodeler conserved from yeast to humans that slides and modifies the composition of nucleosomes on a DNA template /Gerhold et al. 2015). INO80C both is a target of the DRC and physically associates with the downstream effector kinase Rad53/CHK2 (Morrison et al. 2007; Chen et al. 2010). INO80C maps to many transcription start sites in budding yeast yet also contributes to DSB repair and replication fork restart following removal of hydroxyurea (HU) (Gerhold et al. 2015). Interestingly, INO80C can down-regulate transcription by repressing short-lived noncoding RNA at intergenic sites (Alcid and Tsukiyama 2014), possibly by restricting accessibility for RNA polymerase (Xue et al. 2015).

In S-phase cells, transcription and replication compete for the same DNA template, making the transcriptional

(C) 2016 Poli et al. This article is distributed exclusively by Cold Spring Harbor Laboratory Press for the first six months after the full-issue publication date (see http://genesdev.cshlp.org/site/misc/terms.xhtml). After six months, it is available under a Creative Commons License (Attribution-NonCommercial 4.0 International), as described at http:// creativecommons.org/licenses/by-nc/4.0/. 
machinery a frequently encountered obstacle for replication forks (Gonzalez-Aguilera et al. 2008; Azvolinsky et al. 2009). Several mechanisms minimize the negative impact of transcription on DNA replication independently of the checkpoint. For instance, tRNA genes are transiently silenced by Maf1 to promote the passage of replication forks during normal S phase in eukaryotic cells (Nguyen et al. 2010). In addition, both bacteria and eukaryotes express specific DNA helicases such as Sen1/ Senataxin or Aquarius, which remove proteins and/or RNA-DNA hybrids that hinder replication fork progression (Brambati et al. 2015). Under stress, additional mechanisms deal with transcription-replication interference. For example, during osmotic stress, Hogl targets the replication fork protein Mrc1, which leads to a reduced rate of DNA polymerase elongation and a decreased rate of origin firing (Duch et al. 2013). Finally, an activated checkpoint kinase, Mec1/ATR, is thought to release actively transcribed genes from the nuclear periphery upon HU-induced replication stress, although how this affects fork recovery is unclear (Bermejo et al. 2011). The S-phase-specific activation of the checkpoint kinase cascade in budding yeast is specifically compromised in a MEC1 allele, mec1-100 (Cobb et al. 2005).

DNA-RNA hybrids that displace one DNA strand (R loops) form spontaneously at highly transcribed genes (El Hage et al. 2014; Santos-Pereira and Aguilera 2015). These structures appear more frequently in mutants that affect RNA 3' end processing, termination, and nuclear export (Wellinger et al. 2006). Interestingly, such mutants also display high rates of spontaneous DNA damage, impaired replication fork progression, and transcription-associated recombination (Tuduri et al. 2009; Gomez-Gonzalez et al. 2011). The inactivation of the PAF1 complex (PAF1C, composed in budding yeast of Paf1, Rtf1, Leo1, Ctr9, and Cdc73), which travels with RNA polymerase II (RNAPII), leads to an accumulation of $\mathrm{R}$ loops (Wahba et al. 2011), increased recombination rates (Chang et al. 1999), and an elevated sensitivity to replication stress (Dronamraju and Strahl 2014). Consistently, recent work showed that the transcription machinery itself (i.e., RNAPII) seems to contribute to replication fork restart on HU or after base alkylation (Felipe-Abrio et al. 2015).

In unstressed cells, one of the main functions of the conserved PAF1C is to promote transcription elongation by facilitating recruitment of the Set2 histone methyltransferase, which deposits H3K36me3 on gene bodies. Additionally, PAF1C targets Rad6-Bre1 to promoters, allowing H2BK123 monoubiquitylation, which in turn helps the methylation of H3K4 by Set1/COMPASS, a hallmark of active transcription (Wood et al. 2003). Finally, PAF1C is required for proper $3^{\prime}$ end processing and termination of mRNA and snoRNA (Penheiter et al. 2005).

Although transcription-replication interference has been identified as a major source of genome instability in recent years, few studies have examined the role of the Mec1/ATR checkpoint kinase in such conflicts. Using a conditional genetic interaction screen, we explored the link between transcription-mediated blocking of repli- cation forks and Mec1-Ddc2. We found that the S-phase checkpoint-deficient mec1-100 mutant exhibits strong negative genetic interactions with mutations in various subunits of PAF1C or INO80C when cells are exposed to $\mathrm{HU}$, suggesting that these complexes may cooperate to promote survival during replicative stress. Examining this relationship further, we found that PAF1C, like $\mathrm{Mec1}$ and INO80C, is required to promote fork progression and restart under replication stress. In addition, we show that Mec1-Ddc2 can physically associate with INO80C and PAF1C and phosphorylates components of each complex. Since PAF1C is associated with RNAPII, we checked whether PAF1C and INO80C help resolve conflicts between the replication and transcription machineries. This was confirmed by showing that Mec1, INO80C, and PAF1C contribute to the displacement or degradation of RNAPII during HU-induced replication stress. This Mec1-INO80C-PAF1C pathway of RNAPII removal is genetically distinct from the Rad26-Def1-induced RNAPII degradation that occurs in response to UV. Our work suggests that RNAPII is evicted from chromatin and degraded upon collision with replication forks in order to facilitate replication under stress conditions.

\section{Results \\ Conditional synthetic lethality between mec1-100 and loss of INO80C and PAF1C subunits}

The budding yeast mutant mec1-100 carries two point mutations upstream of its kinase domain, which together compromise Rad53 activation on HU in S phase, although mec1-100 is fully competent to activate this same checkpoint cascade in G2/M (Hustedt et al. 2015). In brief, this suggests that mec1-100 loses S-phase-specific interactions that in turn compromise survival on HU. To find this pathway, a high-throughput and conditional genetic interaction screen, termed epistatic miniarray profiling (EMAP), was carried out using the S-phase-specific mec1-100 allele crossed into 1311 strains deleted for nonessential genes implicated in nuclear functions (Hustedt et al. 2015). The growth rates of double mutants were compared with those of single mutants in the presence of 0,20 , and $100 \mathrm{mM} \mathrm{HU}$ in order to identify genetic pathways that are required for survival in the face of replication stress.

A number of mutants showed synergistically negative interactions uniquely in cells exposed to HU (Fig. 1A). Among the strongest negative scores of the screen are genes involved in DNA damage checkpoint signaling (TEL1 and RAD9), targeting to the nuclear pore (NUP133, NUP170, NUP188, and APQ12), and resolution of stalled forks or recombination intermediates (XRS2, MUS81, and RAD59). This is consistent with a requirement for DNA damage tolerance pathways sensu lato to cope with lesions arising from replication fork collapse in a mec1-100 background (Fig. 1A). We also identified strong negative interactions with factors involved in either the ubiquitin/proteasome pathway, chromatin remodeling, or basic RNAPII regulation. Strikingly, the 
A

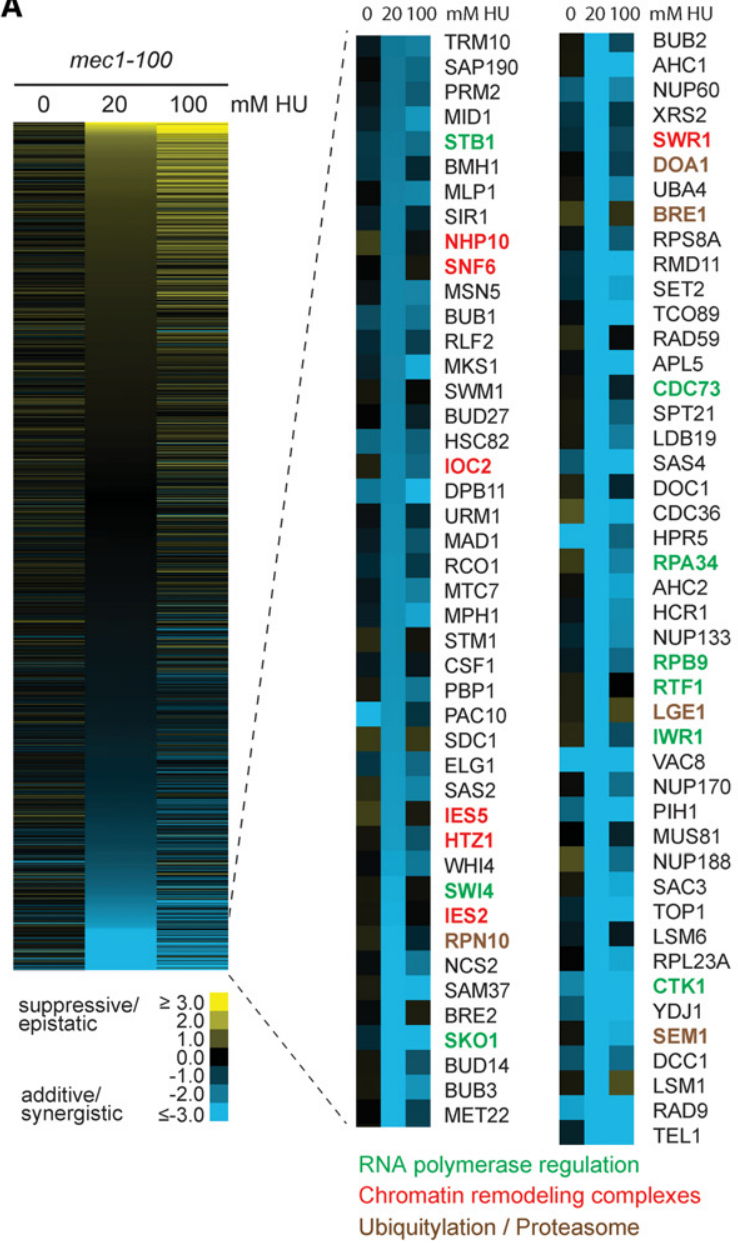

B

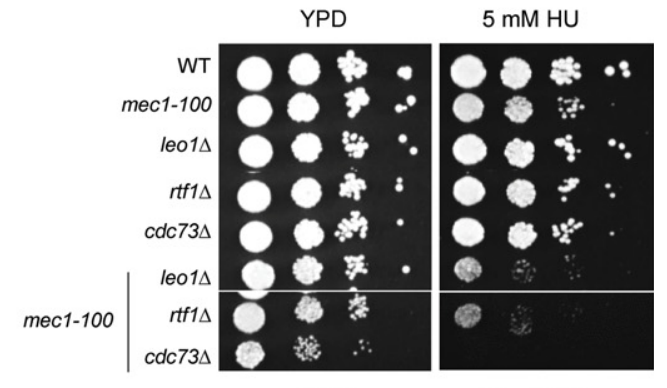

YPD

$10 \mathrm{mM} \mathrm{HU}$
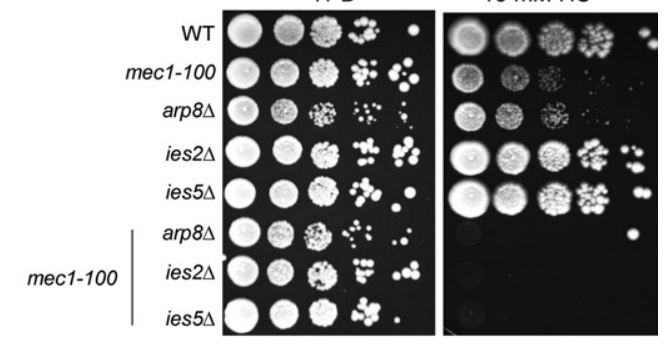

WT $0.00 \div \therefore$

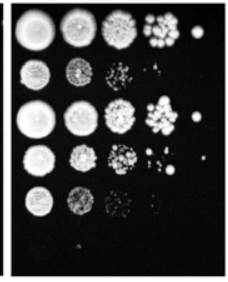

Conditional negative genetic interaction with mec1-100:

Transcription
RPB9
IWR1
CTK1
RTF1
RPA34
STB1
SWI4
SKO1

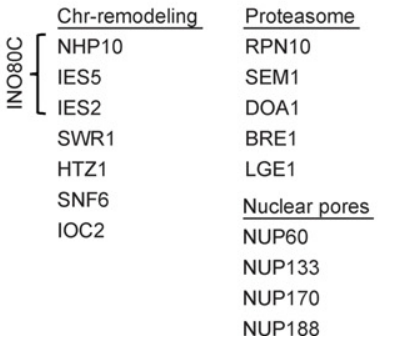

\begin{tabular}{|c|c|}
\hline Histone modification & DNA damage checkpoint \\
\hline RCO1 & DPB11 \\
\hline SAS2 & RAD9 \\
\hline SAS4 & TEL1 \\
\hline \multicolumn{2}{|l|}{ BRE2 } \\
\hline $\mathrm{AHC1}$ & DNA repair \\
\hline SET2 & XRS2 \\
\hline $\mathrm{AHC2}$ & RAD59 \\
\hline BRE1 & HPR5 (SRS2) \\
\hline \multirow[t]{2}{*}{ LGE1 } & MUS81 \\
\hline & MPH1 \\
\hline
\end{tabular}

Figure 1. Mec1 shows strong synergistic lethality with INO80C and PAF1C subunits on HU. $(A)$ Overview of genetic interaction screen (EMAP) (full data in Hustedt et al. 2015). Query strain mec1-100 was combined with 1311 mutant strains, and double mutant growth was scored on 0,20 , and $100 \mathrm{mM}$ HU. Alignment by synthetic lethality scores on $20 \mathrm{mM} \mathrm{HU}$. The bottom panel reveals mutations exhibiting strong negative genetic interactions (blue) with mec1-100 specifically in HU. Transcriptional machinery is indicated in green, chromatin remodeling subunits are shown in red, and the ubiquitin/proteasome pathway is indicated in brown. (B) Confirmation and extension of the negative EMAP interactions between mec1-100 and either PAF1C or INO80C subunits. A fivefold dilution series of cells from exponential YPD cultures of the indicated strains was spotted on YPD with or without the indicated dose of HU.

loss of INO80C subunits (IES5, IES2, and NHP10) or PAF1C subunits (RTF1 and CDC73) was strongly synergistic with mec1-100 on HU but not in its absence. Thus, loss of intact PAF1C and INO80C compromised survival in cells experiencing chronic replication stress.

We confirmed these genetic interactions by crossing the S-phase-specific mec1-100 allele with deletion mutants of various INO80C and PAF1C subunits in a haploid W303 background and scoring for survival under conditions of replication stress. The double mutants were much more sensitive to very low doses of $\mathrm{HU}$ than the single mutants (Fig. 1B) and were also sensitive to slightly higher doses of methyl methanesulfonate (MMS), which alkylates bases and activates the DRC when the lesions encounter a 
replication fork (Supplemental Fig. S1A). Not only was genetic synergy seen with the checkpoint kinase (Mec1), but combining ies2 $\Delta$ (INO80C) with $c d c 73 \Delta$ (PAF1C) was also synergistically lethal on $\mathrm{HU}$ (Fig. 1B). An alignment of the overall profiles of genetic interactions for null alleles in PAF1C and INO80C (Supplemental Fig. S1B) showed a high degree of similarity, a fact that further argues for a common function (Collins et al. 2007). Finally, the triple mutant (mec1-100, ies $2 \Delta$, and $c d c 73 \Delta$ ) was more sensitive to very low $H U$ concentrations than any of the pairwise combinations, suggesting that Mec1, INO80C, and PAF1C may contribute in slightly different ways to cell survival of replication stress (Fig. 1B).

\section{INO80C interacts with Mec1-Ddc2 and PAF1C}

Previous work suggested that Ino80 can be found in higherorder complexes of different sizes (Shen 2004). Given that components of INO80C and Mec1-Ddc2 are necessary for the survival of replication stress, we then asked whether these two complexes physically interact, making use of a strain that carries a Flag epitope inserted after the INO80 ORF (Shen et al. 2000; Tosi et al. 2013) and a 6xHis motif fused to MEC1. We immunoprecipitated INO80C using anti-Flag beads and then tried to separate pure INO80C from that bound to Mec1-6xHis, by immobilized metal affinity chromatography (IMAC) and stepwise imidazole elution. The $100 \mathrm{mM}$ imidazole elution fraction contained Mec1 and its cofactor, Ddc2, along with the 15 subunits of INO80C (Tosi et al. 2013) at roughly equivalent stoichiometry, as judged by Coomassie blue staining of an SDSPAGE gel (Fig. 2A; Supplemental Fig. S2A). In-solution digests and mass spectrometry (MS) confirmed the presence of all subunits of INO80C and Mec1-Ddc2 and revealed an additional five copurifying proteins. Surprisingly, these were the five subunits of PAF1C: Paf1, Rtf1, Ctr9, Leo1, and Cdc73 (Fig. 2A; Supplemental Fig. S2; Jaehning 2010). The fraction of INO80C recovered in complex with Mec1-Ddc2 and PAF1C was $<5 \%$ of the total cellular complement of INO80C, arguing that only a subfraction of the available complex associates stably with the other two complexes (Supplemental Fig. S2). Nonetheless, since the purification protocol involved tandem affinity steps and multiple large-volume washes, it argues for some physical interaction between these multisubunit complexes.

\section{The topology of INO80C-Mec1-PAF1C interactions}

To validate this hypothesis of molecular contact, we combined chemical cross-linking and MS analysis (XL-MS), a method well suited to detect interfaces between low abundance, macromolecular complexes (Leitner et al. 2010). We used the primary amine-specific disuccinimidyl suberate (DSS) that cross-links two lysines at a $\mathrm{C}_{\alpha}-\mathrm{C}_{\alpha}$ span distance of $\leq 30 \AA$ (Tosi et al. 2013). XL-MS results for the INO80C-Mec1-PAF1C fraction revealed largely the same contact sites between the subunits of INO80C as previously detected for purified INO80C (Fig. 2B, green lines; Tosi et al. 2013). In addition, we recovered and validated five cross-links that support the existence of intercomplex interactions: three between Mec1 and INO80C (Fig. 2B, red lines linking Mec1 and Ies2, and Taf14 and the Ino80 helicase SANT-associated [HSA] domain) and two between PAF1C and INO80C (Fig. 2B, red lines linking the Cdc73 C terminus with Arp5 or with the Ino80 RecA2 domain). These sites of contact occur in exposed domains that are available for protein-protein contacts and include the docking site for actin and actin-related proteins; namely, the Ino80 HSA domain (Chen et al. 2013; Tosi et al. 2013). Many additional intracomplex contact sites were also detected and validated in our assay (Fig. 2B, black lines; Supplemental Table S2). Those within INO80C suggest that the remodeler recovered with Mec1-Ddc2 assumes a compact rather than extended conformation (Watanabe et al. 2015). In conclusion, our XL-MS data argue for direct contact between Mec1-INO80C and PAF1-INO80C, although it is not possible to comment on the stability of the interaction. Given that our recovery of cross-linked peptides is nonsaturating, the data do not exclude that PAF1C and Mec1-Ddc2 interact.

\section{Coimmunoprecipitation strategies corroborate intercomplex interactions}

To confirm the intercomplex contacts, we used an alternative two-step affinity purification scheme from a strain in which all three complexes bear epitope tags (Ino80-Flag, Ddc2-HA and Rtf1-PK, and GA-9247). After Flag purification of Ino80, Western blot analysis shows the presence of all three epitope-tagged complexes in the eluate (Fig. 2D), consistent with the results of Figure 2A. We subsequently performed an immunoprecipitation from the Flag eluate for the PK-tagged PAF1C (Rtf1-PK). Whereas the bulk of Ino80-Flag and Ddc2-HA was not recovered with PAF1C (Supplemental Fig. S2B), prolonged exposure of the Western blot of Rtf1-PK immunoprecipitation fractions revealed that Ino80-Flag and Ddc2-HA were recovered at levels well above the control (beads only) (Fig. 2D). Benzonase treatment, which degrades nucleic acids, did not ablate the interaction, although the interaction was slightly stabilized by the addition of ssDNA after benzonase treatment (Supplemental Fig. S2C). To rule out any risk of Flag epitope cross-reactivity, we used a third strain that bears endogenously tagged Ino80-Myc, Ddc2-HA, and Leo1-PK. Using an Arp5-specific antibody to immunoprecipitation from this cell extract, we probed for Myc, HA, and PK tags at the expected molecular weights. Indeed, all complexes were found in an immunoprecipitation of the INO80C subunit Arp5 (Fig. 2E), although Leo1-PK and Ddc2-HA were recovered less efficiently than INO80-Myc.

While there appears to be interaction between INO80C with both Mec1-Ddc2 and PAF1C, it is not possible to postulate a stable trimeric complex. Attempts to estimate the size of higher-order complexes in the Flag immunoprecipitation eluate by Superose 6 gel filtration column suggest that both PAF1C (Rtf1-PK) and Mec1-Ddc2 (Ddc2-HA) elute as more than one higher-order structure, each with some overlap with the broad elution profile of INO80C, in fractions clearly distinct from the void volume (Supplemental Fig. S2D, fractions 27-29 and 
A

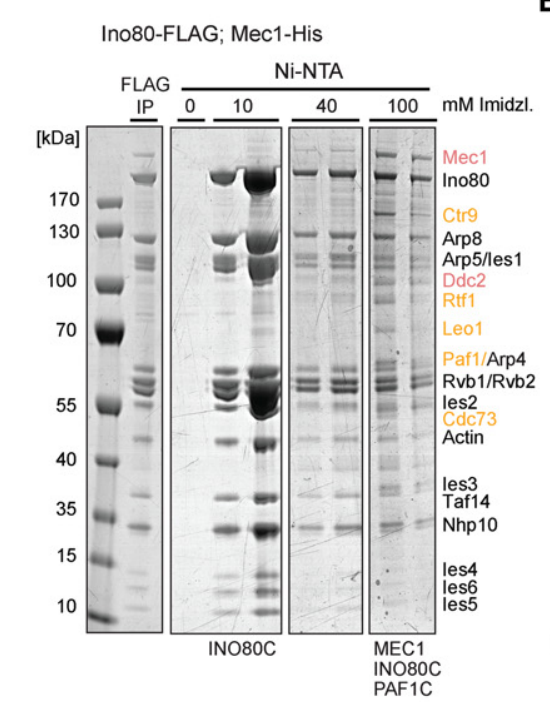

C

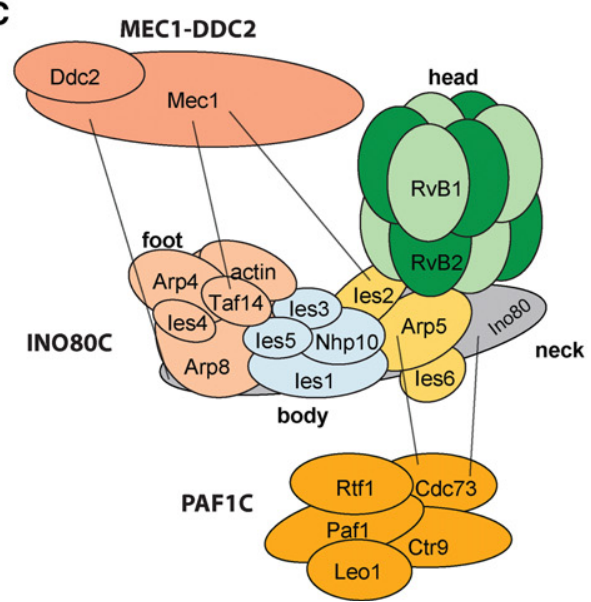

B

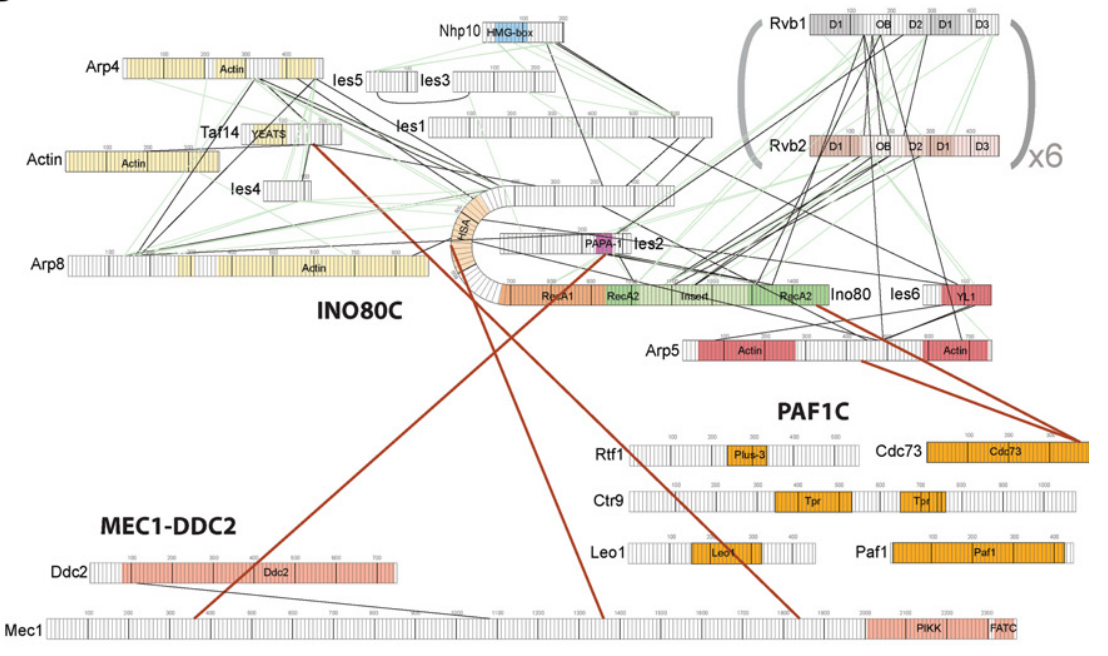

D

E

Ino80-FLAG; Rtf1-PK; Ddc2-HA

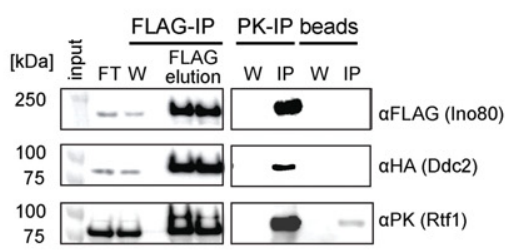

Ino80-Myc; Leo1-PK; Ddc2-HA

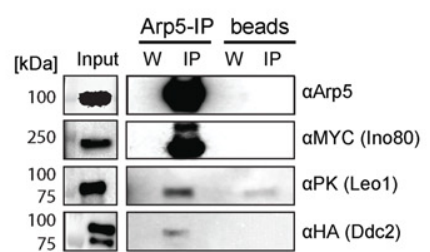

Figure 2. A subset of INO80C interacts with Mec1-Ddc2 and PAF1C. (A) Yeast strain GA8791 was used for the purification of FlagINO80C, followed by Ni-NTA column chromatography. A subset of INO80C coeluted with 6xHis-tagged Mec1 from Ni-NTA, and the final elution contained Mec1-Ddc2 and the complete PAF1C as confirmed by MS. (B) A lysine-specific cross-link interaction map of the fraction containing all three complexes. Light-green lines indicate interprotein cross-links similar to those found in a previous INO80C cross-link map (Tosi et al. 2013). Black (intracomplex) and red (intercomplex) lines show new cross-links found in this study (see Supplemental Table S2). (C) Schematic model of PAF1C and Mec1-Ddc2 association with INO80C based on intercomplex crosslinks. Intra-INO80C contacts may point toward a compact conformation or slightly altered location of the Arp5-Ies6 module. (D) Ino80-Flag purification of a cell extract containing epitope-tagged Ino80-Flag, Ddc2-HA, and Rtf1-PK. Subsequent purification with a PK antibody (V5) contains Ino80-Flag and Ddc2-HA (vs. beads-only control). Western blots for Flag and HA after PK immunoprecipitation were exposed longer than the corresponding PK Western blot. (E) Alternative coimmunoprecipitation to test the interaction between INO80C, PAF1C, and Mec1-Ddc2 using an Arp5 antibody. Signals over background can be detected for Ino80-MYC, Rtf1-PK, and Ddc2-HA in an Arp5 pull-down compared with a beads only control.

21-23). We conclude that these complexes, which show strong genetic interactions on $\mathrm{HU}$, are capable of physical interaction (Fig. 2C).

\section{The INO80-Mec1-PAF1 complexes harbor multiple phosphorylation sites}

The interaction between these complexes is consistent with existing data showing that multiple subunits of INO80 (Ies4, Ies1, and Ino80) and the PAF1C subunit Leo1 are phosphorylation targets of Mec1-Ddc2 on HU
(Hustedt et al. 2015). In addition, the ATPase subunit itself, Ino80, is phosphorylated at residue Ser115 by Rad53 (Fig. 3A; Supplemental Table S3; Bastos de Oliveira et al. 2015). We performed an additional phosphoproteomic analysis to compare modification states between wild-

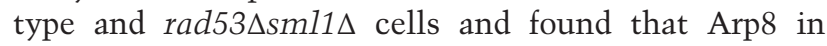
INO80C and Ctr9 in PAF1C are phosphorylated by Rad53 upon exposure to HU as well (Supplemental Table S4). Intriguingly, other phospho-acceptor sites increase, rather than decrease, in the absence of Rad53 on HU (Supplemental Table S4), which may reflect the elevated levels 
A

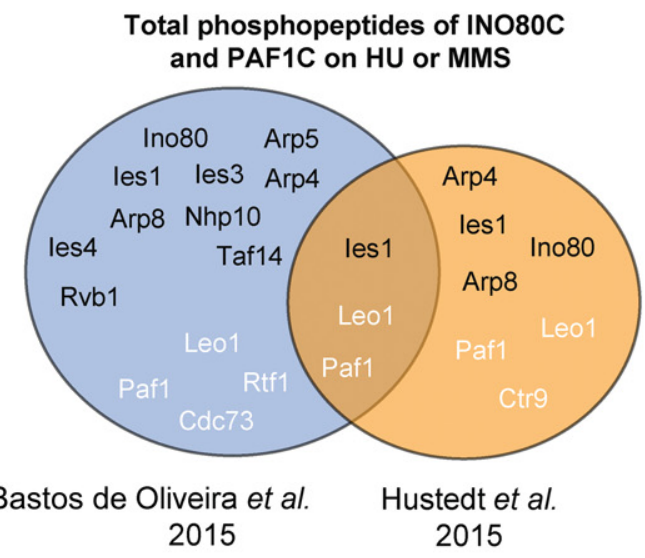

\section{Confirmed Rad53 targets in Confirmed Mec1 targets in INO80C or PAF1C INO80C or PAF1C}

Ino80, Arp8, Ctr9

B

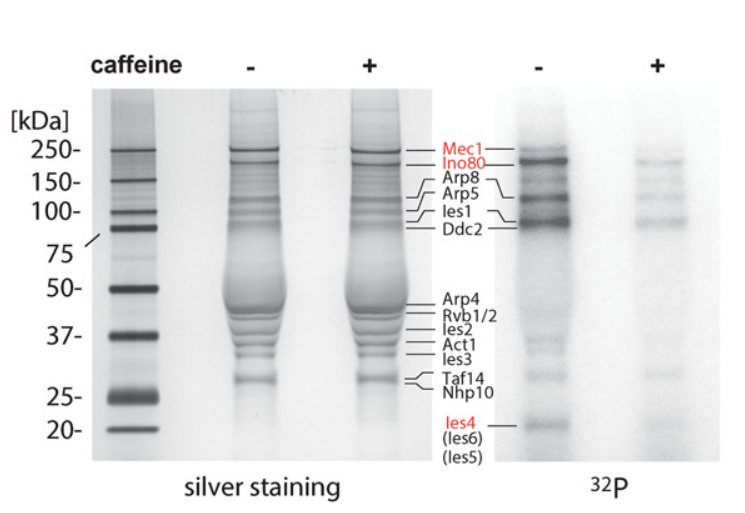

red = validated phosphorylation by MS

\begin{tabular}{|c|c|c|}
\hline $\mathrm{mpl}$ & SU & in vivo detected phospho-acceptor sites \\
\hline \multirow{10}{*}{ 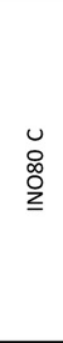 } & $\operatorname{Ino80}$ & S115, S133 \\
\hline & Arp4 & STS $351-353$, T388 \\
\hline & Arp5 & $\mathrm{T} 24, \mathrm{~S} 38$ \\
\hline & Arp8 & S65, S70, SST 233-235 \\
\hline & les1 & S27, S34, T446, T516, SSS 523-525, T567, SS 574-575 \\
\hline & les3 & S211 \\
\hline & les4 & $\mathrm{S} 2, \mathrm{TS} 24-25 ; \mathrm{S} 28$ \\
\hline & Nhp10 & SS 201-202 \\
\hline & Taf14 & ST $136-137$, TT $140-141$ \\
\hline & Rvb1 & SS $15-16$ \\
\hline \multirow{5}{*}{$\begin{array}{l}u \\
\vec{\Delta} \\
\stackrel{\vec{\Delta}}{\Delta}\end{array}$} & Paf1 & $\mathrm{S} 32, \mathrm{~S} 147, \mathrm{~S} 388, \mathrm{~T} 422$ \\
\hline & Rtf1 & $\mathrm{S} 2, \mathrm{~S} 17, \mathrm{~S} 393$ \\
\hline & Leo1 & $\mathrm{S} 105, \mathrm{~S} 132, \mathrm{~S} 339, \mathrm{~S} 358$ \\
\hline & Cdc73 & STTS $147-150$ \\
\hline & $\operatorname{ctr} 9$ & S1015, S1017 \\
\hline
\end{tabular}

C

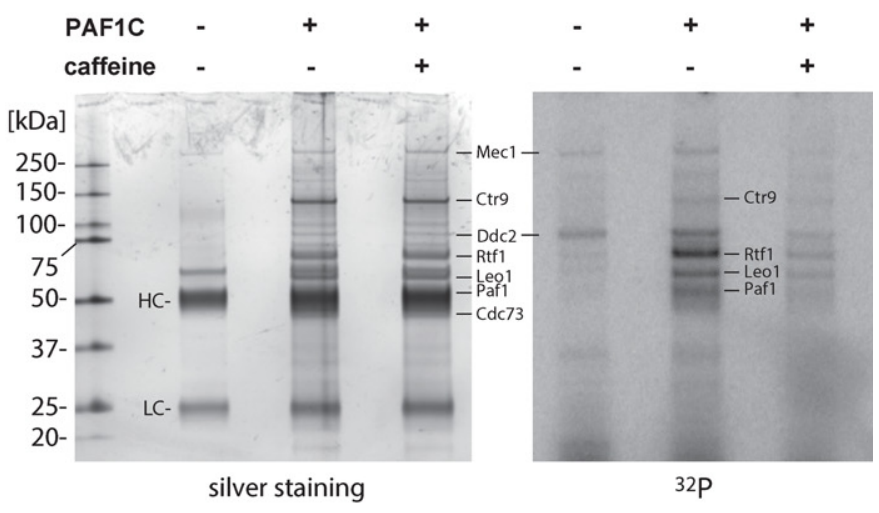

Figure 3. PAF1C and INO80C are phosphorylated by the DRC kinases upon replicative stress. (A) Phosphopeptides identified in either PAF1C or INO80C subunits under replicative stress (HU or MMS) from two recent phosphoproteomic studies (Bastos de Oliveira et al. 2015; Hustedt et al. 2015). Phosphorylation sites include SQ sites, notably in Ies4 (INO80C) and Leo1 (PAF1C) (see Supplemental Table S3). Mec1- and Rad53-dependent phosphorylation on HU is indicated. (B) In vitro Mec1 kinase assay using ${ }^{32} \mathrm{P}$ on purified INO80C subunits identified by molecular weights (see Fig. 2). The Ni-NTA elution of purified Mec1-Ddc2, INO80C, and PAF1C was incubated in the presence of $\gamma^{-32} \mathrm{P}$-ATP with or without $30 \mathrm{mM}$ caffeine for $30 \mathrm{~min}$ at $30^{\circ} \mathrm{C}$. In a separate experiment, MS analysis allowed the identification of Mec1, Ino80, and Ies4 caffeine-dependent phosphopeptides (shown in red) after the kinase assay. (C) In vitro Mec1 kinase assay on purified PAF1C. Myc-tagged Mec1 was immunoprecipitated and incubated alone or with purified PAF1C as in B. Light (LC) and heavy (HC) IgG chains are indicated.

of fork-associated damage that occur in the absence of Rad53, which in turn increases Mec1 and/or Tell activity.

To validate these phosphoproteomic findings, we tested whether INO80C subunits are modified in vitro by the copurifying Mec1-Ddc2 complex (Mallory and Petes 2000). We incubated the INO80C-Mec1 fraction isolated by Flag tag purification with $\gamma^{-32} \mathrm{P}$-ATP in the presence or absence of caffeine. Analysis by SDS-PAGE and radiography indicates that bands comigrating with Mec1, Ino80, Ddc2, and Ies4-bona fide targets of Mec1-Ddc2 (Paciotti et al. 2000; Hustedt et al. 2015)-incorporate ${ }^{32} \mathrm{P}$ in a caffeine-sensitive manner (Fig. 3B). MS confirmed caffeine-sensitive enrichment for SQ/TQ acceptor sites on
Mec1 (Ser38), Ino80 (Ser51, Thr568), and Ies4 (Ser2) after incubation with ATP (Fig. 3B, labeled in red). The other bands incorporating $\gamma^{-32} \mathrm{P}$ in a caffeine-sensitive manner in the complex could not be unambiguously assigned based on migration alone (Fig. 3B) but appear to correspond to Arp5, Arp8, or Ies2. We also confirmed that subunits of PAF1C are specifically modified by Mec1-Ddc2 by incubating $\mathrm{Mecl}$ recovered by immunoprecipitation with PAF1C isolated from yeast. Besides the kinase subunits themselves, Rtf1, Leo1, and Paf1 appear to acquire caffeine-sensitive modification (Fig. 3C). Finally, the purified INO80C, PAF1C, Mec1-Ddc2 fraction was shown to retain caffeine-sensitive kinase activity for a well- 
characterized Mec1-Ddc2 target peptide, PHAS-1 (Supplemental Fig. S2E; Mallory and Petes 2000). We conclude not only that INO80C and PAF1C are modified in response to replication stress but that multiple subunits are modified by Mec1-Ddc2 in vitro. However, given the multiplicity of phosphoacceptor sites, it is difficult to ascertain the functional relevance of such modifications.

\section{Paf1 promotes replication under $H U$-induced stress}

Whereas our initial analysis detected strong genetic interactions necessary for the survival of replication stress between null alleles of single subunits of these three complexes, strains lacking only Leo1, Rtf1, or Cdc73 did not show significant sensitivity to 5 or $10 \mathrm{mM} \mathrm{HU}$ (Fig. 1B). To see whether this insensitivity reflected the degree of stress imposed, we checked the growth of these three individual mutants along with paf1 $1 \Delta$ on plates containing either $50 \mathrm{mM}$ HU or $0.01 \%$ MMS. At higher levels of damage, deletion of any single subunit of the RNAPII-associated factor PAF1C indeed sensitized cells to replication stress (Fig. 4A).

Given that Mec1 and INO80C not only are required for the survival of chronic exposure to HU but also contribute to replication fork progression after brief exposure to acute levels of HU (Branzei and Foiani 2010; Gerhold et al. 2015), we examined whether this was also true for PAF1C. We compared the resumption of replication in paf1 1 and mec1-100 mutants by FACS following a 2-h exposure to $0.2 \mathrm{M} \mathrm{HU}$. Although wild-type cells resumed replication and fully duplicated their genomes by 75 min after HU removal, paf1 $\Delta$ cells showed a severe delay in the recovery process, much like the mec1-100 mutant (Fig. 4B). To confirm that this delay in replication was due to impaired fork progression, we monitored DNA synthesis at the level of individual replication forks using DNA combing (Fig. 4C). Consistent with the FACS analysis, we found that replication forks synthesize shorter stretches in paf1s versus wild-type cells in both early S phase (90 min in HU; 12 vs. 13 kb) (Fig. 4D,E) and mid-S phase (180 min in HU; 25.5 kb vs. 32.5 kb) (Fig. 4D,E). Taken together, this confirms that PAF1C itself impacts replication fork progression in the face of replication stress.

\section{PAF1C is removed from chromatin upon HU treatment in a Mec1-dependent manner}

To account for this surprising effect of an RNAPII cofactor on DNA polymerase progression, we first asked whether PAF1C relocates to replication sites in HU-treated cells, as was observed for Sen1 (Alzu et al. 2012). However, using ChIP-qPCR (chromatin immunoprecipitaton [ChIP] combined with quantitative PCR [qPCR]), we could not detect any significant enrichment of PAF1C at stalled forks, whether at early firing origins (ARS305 and 306) or late firing origins (ARS809 and ARS911) (Supplemental Fig. S3G). Previous work demonstrated that PAF1C associates with the elongating RNAPII in asynchronously growing cells (Wade et al. 1996), dropping off upstream of the polyadenylation site (Mayer et al. 2010), yet no one had tracked PAF1C in cells synchronously enduring replicative stress. Therefore, we examined the chromatin association of PAF1C in cells synchronized in G1 with those released into early $S$ phase on HU using ChIP-qPCR at two loci known to bind PAF1C in asynchronous conditions. ChIP for the PAF1C subunit Leol showed that a surprisingly large fraction of PAF1C was lost from $P Y K 1$ and YEF3 genes after $\mathrm{HU}$ treatment in wild-type cells (Fig. 4F). As phosphorylation of Leol is deficient in a mec1-100 strain (Hustedt et al. 2015), we tested whether the mec1100 allele affects PAF1C eviction by ChIP-qPCR for Leo1 in G1 and HU, comparing wild-type and mec1-100 cells. We found that Leol removal was compromised in mec1-100 cells, suggesting that its release from chromatin requires an S-phase-specific function of Mec1 (Fig. 4F). This correlates with a mec1-100-sensitive phosphorylation event in Leol on HU (Hustedt et al. 2015).

RNAPII disengages from chromatin during HU-induced replicative stress

Transcription is known to hinder the progression of replication forks (Felipe-Abrio et al. 2015), and this might be further enhanced when replication forks reduce speed or are unable to progress in the absence of a functional DRC (Poli et al. 2012). Since PAF1C disengages from chromatin upon HU treatment, we reasoned that the transcription machinery itself may also be removed from chromatin. To address this possibility, we performed chromatin fractionation (Fig. 5A) to measure the global level of RNAPII associated to chromatin in synchronized wild-type cells exposed to HU. When compared with the level detected in G1-phase cells, we found that the level of RNAPII on chromatin is reduced to $55 \%$ (Fig. 5B,D). In order to accommodate variations in RNAPII levels that might arise from the cell cycle (G1 vs. S phase), we compared RNAPII levels in a-factor-arrested cells (G1) with an early time point in $\mathrm{S}$ phase without $\mathrm{HU}$ (released for $20 \mathrm{~min}$ ). In unperturbed S-phase cells, on the other hand, we observed that level of chromatin-bound RNAPII increases over that in G1 (Supplemental Fig. S3A,B). Thus, we conclude that RNAPII is selectively evicted from chromatin when S-phase cells are exposed to replication stress. Finally, using specific phospho-antibodies, we checked whether the initiating (Rpb1 Ser5-P) or elongating (Rpb1 Ser2-P) form of RNAPII was preferentially evicted from chromatin upon HU-induced stress. Only the initiating RNAPII (Ser5-P) seems to be evicted from chromatin on HU (Supplemental Fig. S3B,C), consistent with the fact that INO80C preferentially binds near sites of transcriptional initiation (Shimada et al. 2008) and the fact that the steady-state levels of mRNA of the loci tested do not change as a reflection of this eviction event (Supplemental Fig. S4I).

\section{RNAPII eviction from chromatin during replication stress depends on Mec1}

Given that Mec1 was required for PAF1C removal from chromatin during HU-induced stress (Fig. 4), we next tested whether Mecl is required for RNAPII eviction. We 
Poli et al.

A
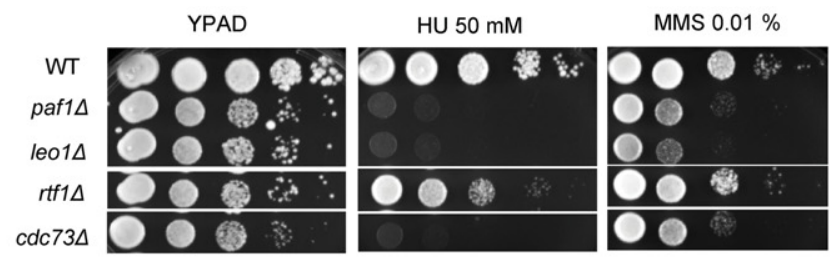

B

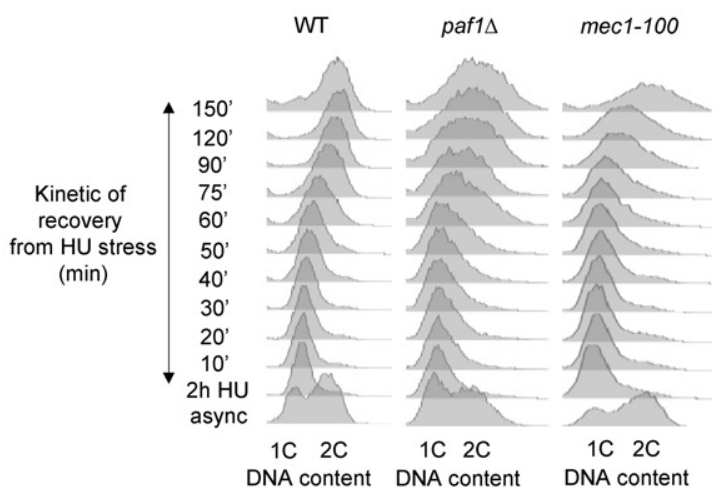

C

D

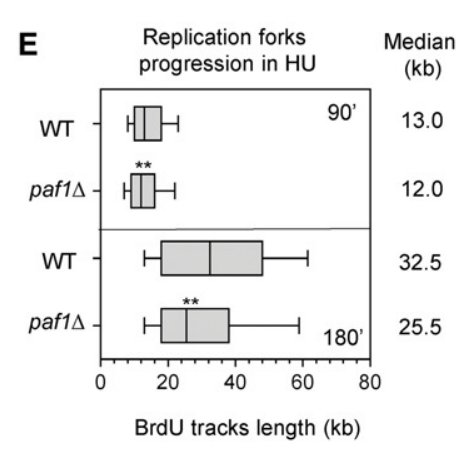

$\mathbf{F}$
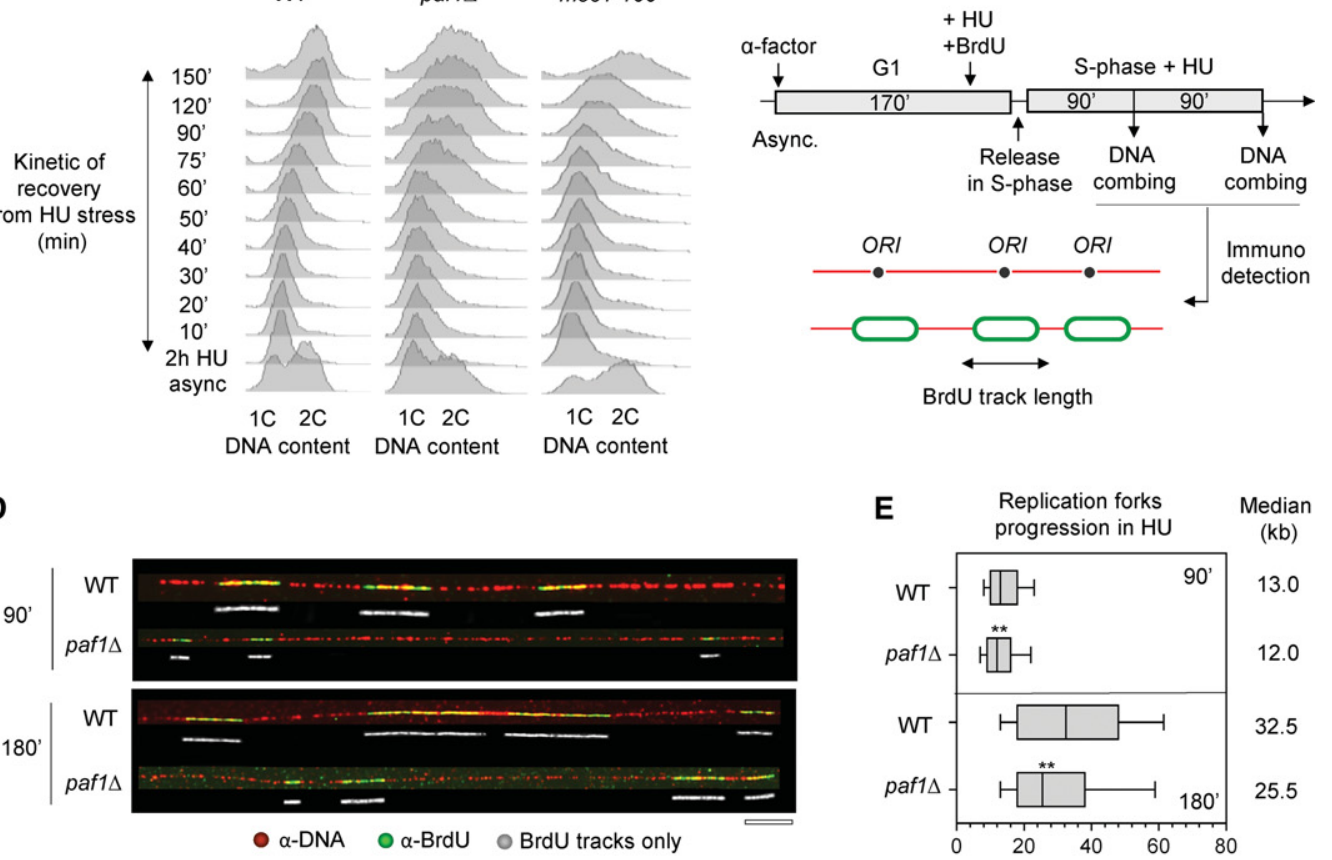

$\begin{array}{ccc}1 \mathrm{C} 2 \mathrm{C} & 1 \mathrm{C} 2 \mathrm{C} & 1 \mathrm{C} 2 \mathrm{C} \\ \text { DNA content } & \text { DNA content } & \text { DNA content }\end{array}$
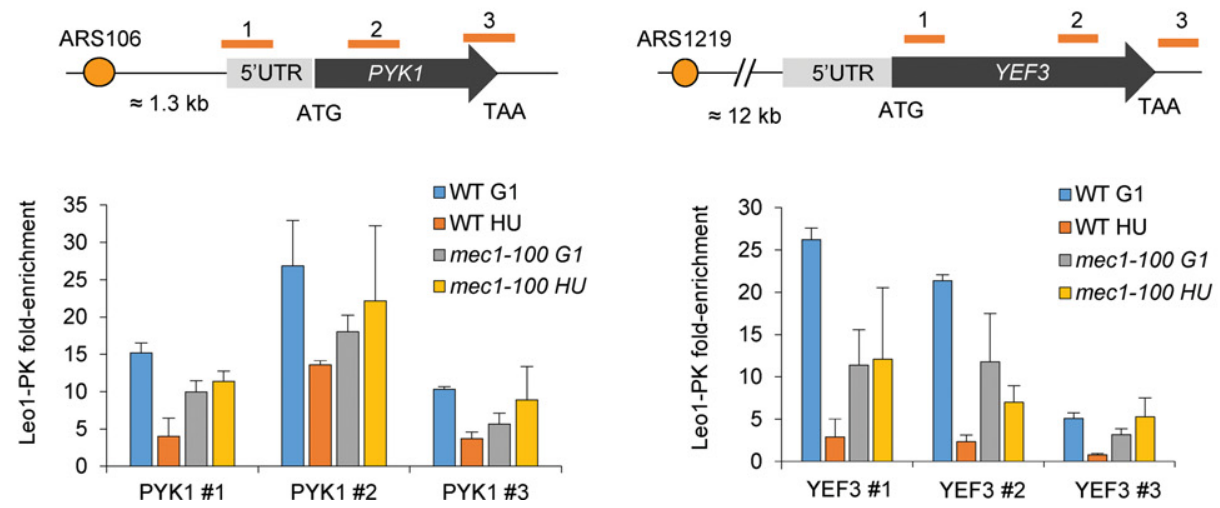

Figure 4. PAF1C promotes replication under HU-induced replication stress. (A) A defined number of exponentially growing cells (fivefold dilutions) was spotted on YPD or YPD plates containing the indicated dose of HU. (B) Flow cytometry analysis of DNA content. Asynchronous cells were treated for $2 \mathrm{~h}$ with $200 \mathrm{mM} \mathrm{HU}$. After HU removal, recovery from replication stress was monitored (time in minutes). $(C, D)$ Analysis of replication fork progression at the single-molecule level by DNA combing. $(C)$ Schematic of the experimental procedure: Exponentially growing cells were synchronized in G1 with a factor and released in S phase in the presence of $200 \mathrm{mM} H U$. Newly replicated DNA was labeled with BrdU for 90 and $180 \mathrm{~min}$. (D) Representative images of DNA fibers are presented. (Green) BrdU; (red) DNA; (gray) BrdU tracks only. Bar, $10 \mathrm{~kb}$. (E) The graph depicts the distribution of BrdU track lengths. Box and whiskers indicate 25th-75th and 10th-90th percentiles, respectively. Median BrdU track length is indicated in kilobases. Asterisks indicate the $P$-value of the statistical test (Mann-Whitney rank sum $t$-test). ( $\left.{ }^{* *}\right) P$-value $<0.01$. (F) ChIP-qPCR (chromatin immunoprecipitaton [ChIP] combined with quantitative PCR [qPCR]) was performed on Leo1-3PK (PAF1C subunit) in G1 and after $60 \mathrm{~min}$ in S phase $+0.2 \mathrm{M}$ HU. Leo1 enrichment was quantified at two loci (PYK1 and YEF3). Data are expressed as fold enrichment. Error bars represent SEM of two individual experiments. 
A

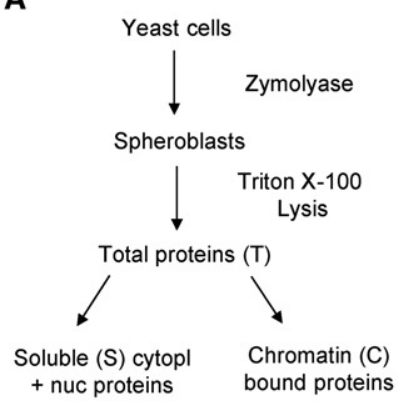

B

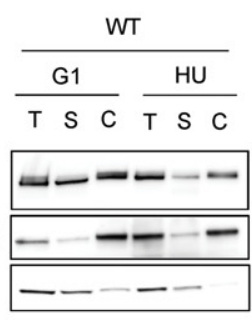

$(73.4 \%)$

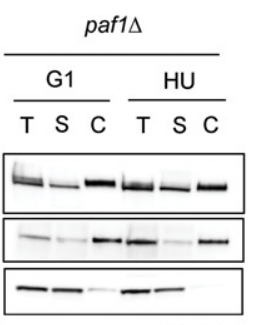

$(102 \%)$
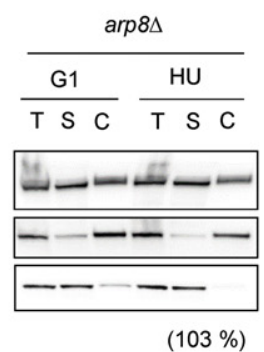

C

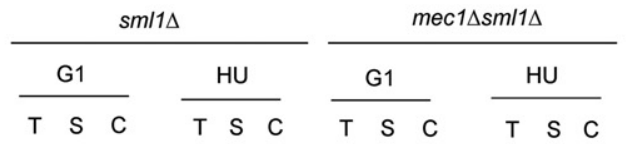

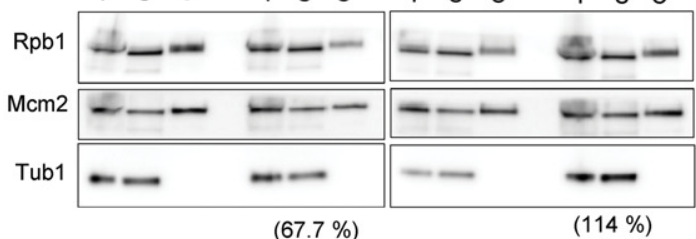

E
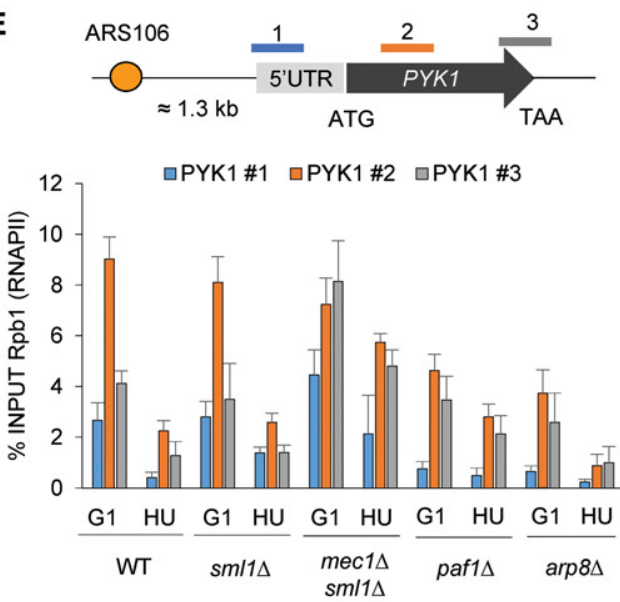

$\mathbf{F}$

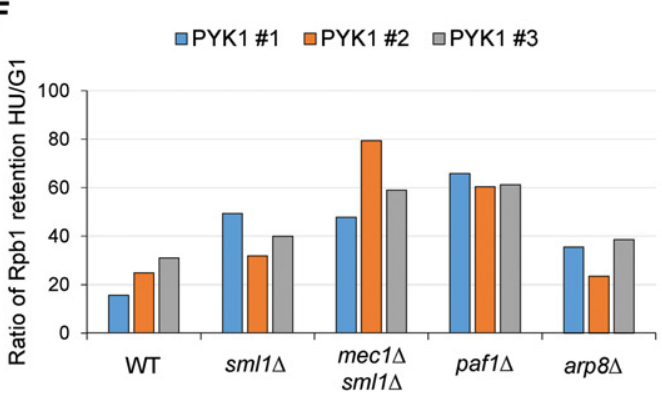

D

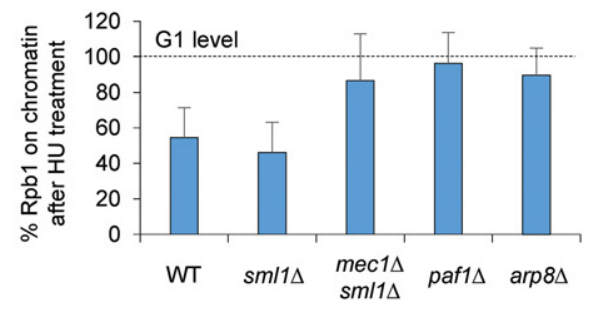

G
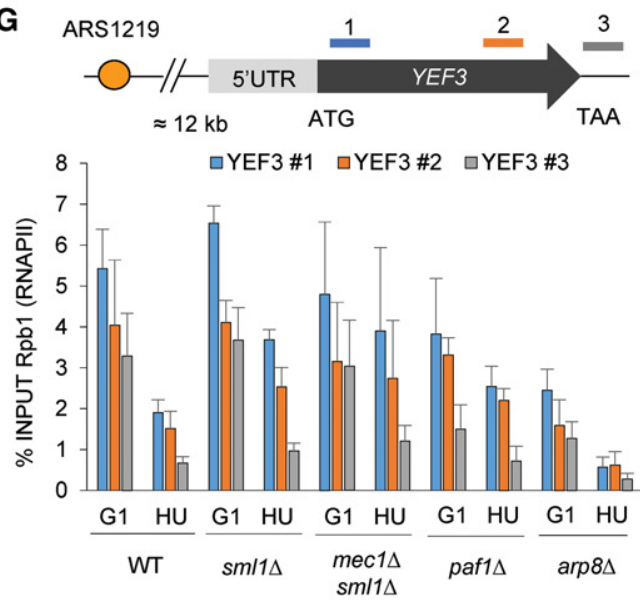

H

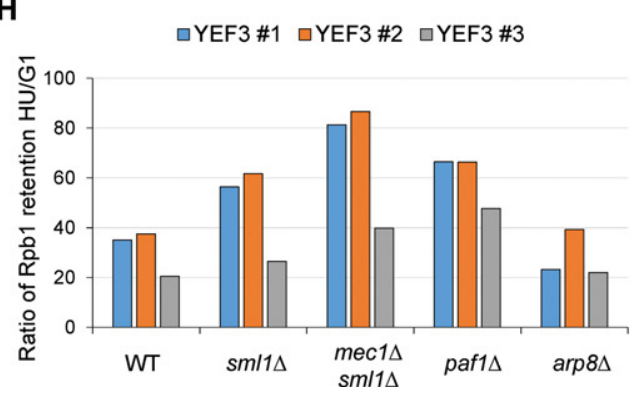

Figure 5. RNAPII is rapidly removed from chromatin following HU treatment in S phase. $(A-D)$ RNAPII association to chromatin was analyzed using chromatin fractionation. $(A)$ Schematic of the experimental procedure: Exponentially growing cells were synchronized in G1 with a factor and released for $60 \mathrm{~min}$ in S phase in the presence of $200 \mathrm{mM} \mathrm{HU}$. (T) Total protein extracts; (S) soluble proteins; (C) chromatin-associated proteins. $(B, C)$ Fractions were subjected to SDS-PAGE followed by immunoblotting with anti-Rpb1-CTD (RNAPII), anti-Mcm2, and anti-tubulin (Tub1) antibodies. Mcm2 was used as a loading control for chromatin, whereas Tub1 was used as a loading control for total protein. $(D)$ Quantification of Rpb1 retained on chromatin after HU treatment, expressed as a percentage over the starting level in G1 (dotted line). Error bars represent SD of at least two individual experiments for each mutant. (E-H) RNAPII retention on chromatin in HU was assessed by ChIP-qPCR by measuring the level of Rpb1 both in G1 and after 60 min in S phase $+0.2 \mathrm{M}$ HU. Rpb1 enrichment was quantified at two loci: PYK1 $(E)$ and YEF3 $(G)$. Data are expressed as a percentage of input. Error bars represent SEM of three individual experiments. $(F, H)$ Graphs depict the mean percentage of Rpb1 kept on chromatin after HU treatment, calculated as a ratio HU/G1 level derived from the mean ChIP-qPCR values. 
monitored chromatin-bound RNAPII in G1-arrested versus HU-arrested S-phase cells lacking the Mec1 checkpoint kinase (Fig. 5C,D). Indeed, whereas RNAPII eviction was $\sim 45 \%$ in wild-type cells, we scored only $13 \%$ eviction in isogenic mec1 $1 \Delta$ sml1 $\Delta$ cells. The mec $1 \Delta$ strain depends on SML1 deletion for viability; thus, as a control, we checked for RNAPII removal in response to $\mathrm{HU}$ in a $s m l 1 \Delta$ strain. An isogenic sml1s strain resembled the wild-type cells for RNAPII eviction from chromatin upon exposure to $\mathrm{HU}$ (Fig. 5C,D). To discriminate between a direct role of $\mathrm{Mec} 1$ and the involvement of downstream effectors of the DRC, we assessed RNAPII levels after $\mathrm{HU}$ treatment in a rad53 $\Delta \mathrm{sm} 11 \Delta$ strain. Interestingly, RNAPII eviction occurs in the absence of Rad53 (Supplemental Fig. S3E). We conclude that Mec1, and not the downstream DRC, controls RNAPII removal.

\section{PAF1C and INO80C contribute to HU-dependent RNAPII removal}

Because Mec1-Ddc2 directly and specifically modifies PAF1C and INO80C subunits on HU, exhibits negative genetic interactions, and coimmunoprecipitates with both complexes, we reasoned that PAF1C and INO80C might also control RNAPII engagement, thereby increasing tolerance of replication stress by reducing replication fork collision with active transcription. To test this, chromatin fractionation was used to measure RNAPII levels after exposure to $\mathrm{HU}$ in paf1 $\Delta$ and $\operatorname{arp} 8 \Delta$ strains. Similar to the results obtained for mec $1 \Delta \operatorname{sm} 11 \Delta$, both paf1 $\Delta$ and arp $8 \Delta$ mutants failed to reduce the level of chromatinassociated RNAPII after the induction of replicative stress (10\% RNAPII eviction in arp $8 \Delta$ vs. $13 \%$ and $4 \%$ in mec $1 \Delta s m l 1 \Delta$ and paf1 $\Delta$ strains, respectively) (Fig. 5B,D). Since paf1 $\Delta$ cells exhibit a delay in the activation of Rad53 upon HU treatment (Supplemental Fig. S3F), we confirmed the RNAPII eviction defect in $c d c 73 \Delta$, another mutant of the PAF1C that does not interfere with Rad53 phosphorylation in HU (Supplemental Fig. S3D-F). Thus, the PAF1C effect is not due to indirect ablation of the DRC. It is also possible that the PAF1C leads indirectly to RNAPII eviction by promoting expression of an HUinduced factor. We therefore scored RNAPII eviction in wild-type cells unable to synthesize new proteins by treating them with cycloheximide. HU-induced RNAPII removal occurs identically in the absence of de novo protein synthesis (Supplemental Fig. S3E,D), supporting the notion that PAF1C acts directly in the eviction process. Taken together, our data argue that the HU-induced removal of RNAPII from chromatin depends on not only Mec1 but also two of its substrates: PAF1C and INO80C.

\section{RNAPII eviction at sites of transcription-replication interference}

To determine whether RNAPII eviction during replicative stress is general or due to specific fork-RNAPII collision, we measured RNAPII at various sites of replication-transcription interference by ChIP-qPCR. We first examined several highly transcribed loci that are known to bind
PAF1C (Mayer et al. 2010) and sit close to early and efficient origins of replication. PYK1 and YEF3 have a codirectional configuration for transcription and replication polymerase movement, while PDC1 and snR13 transcribe into the fork. Using available BrdU-IP-chip maps (Poli et al. 2012), we were able to precisely position the distance covered by individual replication forks at the studied loci (Supplemental Fig. S4A-H). While PDC1, PYK1, and snR13-TRS31 are fully replicated, YEF3 is located $\sim 10 \mathrm{~kb}$ from the replication machinery after $60 \mathrm{~min}$ in HU (Supplemental Fig. S4B). Using RT-qPCR, we confirmed that these loci do not show cell cycle or HU stress-induced changes in steady-state mRNA levels (Supplemental Fig. S4I), unlike genes that are specifically downregulated in S phase, like FUS1 (Supplemental Fig. S5I).

Quantitative ChIP-qPCR allowed us to compare the amount of RNAPII engaged at these loci in G1 as compared with the level after 60 min on HU. In a wild-type strain, we scored an $\sim 75 \%$ decrease of RNAPII association at PYK1, PDC1, and snR13-TRS31 and a 60\% drop at YEF3 even though this gene is not yet replicated at the time point analyzed (Fig. 5E-H; Supplemental Fig. S4). Given that RNAPII levels were reduced at a locus prior to its replication, we extended our analysis to other loci with the aim of discriminating between a cis effect mediated by direct collision with the replication machinery and a global effect generated by the replication stress. First, we could exclude that all loci show a similar drop in RNAPII: We did not observe any change in RNAPII level at MUP1, a gene located far away from any replication forks and whose transcription is not affected by the HU treatment (Supplemental Figs. S4, S5E,I). For a locus that is specifically repressed by the HU treatment, FUS1, we could not detect RNAPII at all (Supplemental Figs. S4, S5H,I). In contrast, we detected de novo recruitment of RNAPII at RNR4, a highly HU-induced gene localized far from replication sites (Supplemental Figs. S4, S5F,I). Collectively, these data support the view that HU-induced replicative stress does not lead to a global removal of RNAPII, consistent with the fact that some genes are actually induced by such stress. The effect occurs primarily at genes that have encountered the replication machinery, yet it seems to propagate to some nearby loci (e.g., YEF3).

To examine further this notion of propagation to genes near predicted sites of replication fork-RNAPII collision, we chose two genes-BAP2 and HIS4-that are found close to replication forks ( 1 and $1.1 \mathrm{~kb}$, respectively) (Supplemental Fig. S4G,H) but are not yet replicated after 60 min on HU, much like YEF3. We detected a drop in RNAPII levels at BAP2, much like YEF3, but not at HIS4 (Supplemental Fig. S5G,H). This may reflect the efficiency of firing of the nearby origin, the efficiency of transcription, or the specific character of the transcription factors bound at this promoter. Indeed, HIS 4 transcripts are at least fivefold lower than those of the other loci (Supplemental Fig. S5I). These results argue that RNAPII removal occurs in cis at sites at which transcription and replication machineries collide but also suggest that it can propagate to a subset of nearby loci. 
Mec1 and PAF1C are required to evict RNAPII at sites of replication fork collision

We next tested how Mec1, PAF1C, or INO80C contributes to RNAPII removal at these specific loci. Consistent with our previous results, paf1 $\Delta$ cells failed to evict RNAPII on HU at PYK1 (Fig. 5E,F), YEF3 (Fig. 5G,H), PDC1 (Supplemental Fig. S5A,B), and, to a lesser extent, snR13-TRS31 (Supplemental Fig. S5C,D). Surprisingly, we did not detect differences between $\operatorname{arp} 8 \Delta$ and wildtype cells at the four loci tested (Fig. 5E-H; Supplemental Fig. S5A-D), although we did detect compromised eviction on a global level in arp $8 \Delta$ cells (Fig. 5B,D). This may indicate that INO80C functions only at a subset of transcribed loci or within a specific genomic context.

Our ChIP analysis showed that loss of Mecl compromises the removal of RNAPII at PYK1 and YEF3 (Fig. 5E-H) but not at PDC1 and snR13-TRS31 (Supplemental Fig. S5AD). Interestingly, both PYK1 and YEF3 are transcribed in the same direction as the replication fork, while PDC1 and $s n R 13-T R S 31$ are transcribed against the fork. Intriguingly, previous work implicated Sen 1 specifically in the maintenance of replication fork stability only in this latter configuration (Alzu et al. 2012). We note that cells bearing the mec1-100 allele, like rad53 $\Delta s m l 1 \Delta$ or sml1 $\Delta$ alone, are still able to remove RNAPII on HU at these specific loci (Supplemental Fig. S6). This is not surprising, given that mec1-100 retains catalytic activity and primarily compromises Rad53 activation during the DRC. Our data argue that Mec1 plays a direct role in RNAPII eviction during replicative stress when transcription and replication are codirectional.

\section{RNAPII is degraded upon HU-induced replication stress}

Stalled RNAPII after UV irradiation is actively degraded by the transcription-coupled nucleotide excision repair (TC-NER) machinery (Somesh et al. 2005). Since transcription-replication conflicts are likely to stall both machineries, we reasoned that RNAPII degradation could be responsible for the reduced levels of RNAPII found on transcribed genes that encounter replication forks. To test this hypothesis, we monitored the total level of Rpb1 in a time-course experiment using wild-type cells synchronized in G1 and then released into a replicationstressed S phase. Total levels of Rpb1 were rapidly and significantly reduced after the release from a factor into medium containing $\mathrm{HU}$, dropping to $\sim 65 \%$ of the G1 level (Fig. 6A,B) at a population scale. We could also provoke Rpb1 degradation by directly treating asynchronously growing wild-type cells with $0.2 \mathrm{M} \mathrm{HU}$, although, as expected, the response occurs less synchronously (Supplemental Fig. S7A). To rule out an alternative effect of $\mathrm{HU}$ (other than replication fork progression), we treated cells blocked in G1 with HU. As expected, HU in the absence of replication did not reduce total levels of RNAPII (Supplemental Fig. S7B,C), allowing us to conclude that replication itself is required for the observed reduction in RNAPII levels.
It has recently been proposed that damage-stalled RNAPII is stably associated to chromatin and requires both INO80 and the proteasome to be removed from the DNA template (Lafon et al. 2015). To investigate whether the RNAPII degradation that we scored upon replicative stress is proteasome-mediated, we measured RNAPII association to chromatin after HU treatment in a pre1-1 pre2-2 strain, which has severe defects in the 26S-mediated proteolysis. Interestingly, in cells lacking a functional proteasome machinery, RNAPII removal from chromatin is inhibited (Fig. 6C). This suggests that RNAPII removal and degradation during replicative stress are coupled.

Finally, we sought to confirm our population-based results for RNAPII degradation on a single-cell level by using high-resolution microscopy to follow the intensity of both RNAPII (Rpb1-eGFP) and PAF1C (Paf1-Ruby2) during exposure to HU. Consistent with Western blot analysis, we show that Rpb1 levels in individual cells progressively decrease as cells endure HU-induced replication stress, reaching $\sim 65 \%$ of the G1 level after $150 \mathrm{~min}$ in $\mathrm{HU}$ (Fig. 6D,E). Again, Rpb1 degradation does not happen in G1-phase cells treated with HU (Fig. 6E). Unlike Rpb1, Paf1 levels do not decrease significantly upon HUinduced replicative stress (Fig. 6F).

Based on the observation that eviction and degradation of RNAPII are coupled upon HU treatment and the fact that both Mec1 and INO80 deal with damage-stalled RNAPII (Taschner et al. 2010; Lafon et al. 2015), we next checked whether Mec1 was required for RNAPII degradation upon HU treatment. Indeed, consistent with its role in RNAPII removal, Mec1 was also required for degradation of RNAPII in HU (Fig. 6A,B; Supplemental Fig. S7A), although the mec1-100 did not compromise RNAPII degradation (Supplemental Fig. S6I,J). Finally, we also checked whether RNAPII degradation requires PAF1C or INO80C integrity. Again, both the paf1 $\Delta$ and arp $8 \Delta$ mutants compromised RNAPII degradation on HU (Fig. 6A,B). Thus, a fraction of RNAPII is degraded specifically in S-phase cells undergoing replication stress through a pathway that depends on Mec1, PAF1C, and INO80C.

Degradation of RNAPII on HU differs from UV-induced degradation

In the presence of UV-induced DNA damage, RNAPII degradation is a last-resort mechanism for survival, induced by Def1 through polyubiquitination and counteracted by $\operatorname{Rad} 26 / \mathrm{CSB}$ (Gaillard and Aguilera 2013). Deleting $R A D 26$ in a def1 $\Delta$ strain restores normal degradation of RNAPII in response to UV (Woudstra et al. 2002) and partially rescues mec1 $1 \Delta \operatorname{sml1\Delta }$ sensitivity to UV (Taschner et al. 2010). To determine whether there is overlap between the Mec1-PAF1C-INO80C eviction that we observed on HU and the Mec1-Rad26-Def1 pathway on $\mathrm{UV}$, we first screened for genetic synergy or epistasis between mec1 $1 \Delta$ sml1s, paf1 $\Delta$, or arp $8 \Delta$ with rad26 $\Delta$ under chronic exposure to HU. The growth of rad26 $\Delta$ cells on HU was indistinguishable from that of wild-type cells (Supplemental Fig. S7D), and there was no synergy or suppression of HU sensitivity when rad26 $\Delta$ was coupled with 
Poli et al.

A

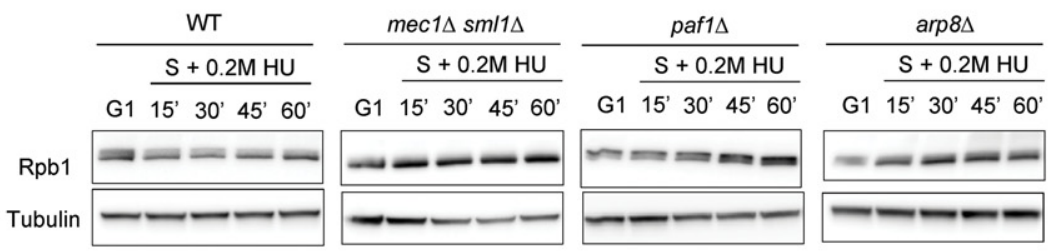

B

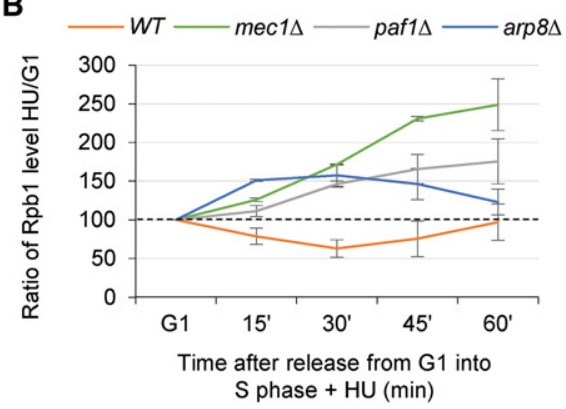

D

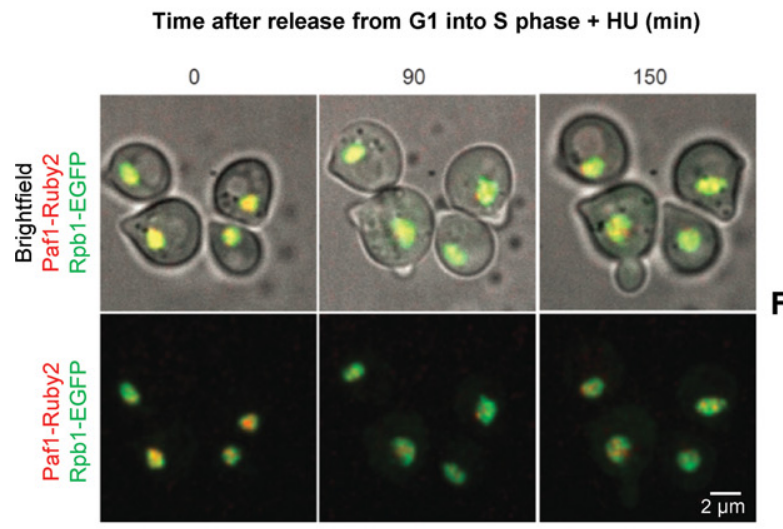

G

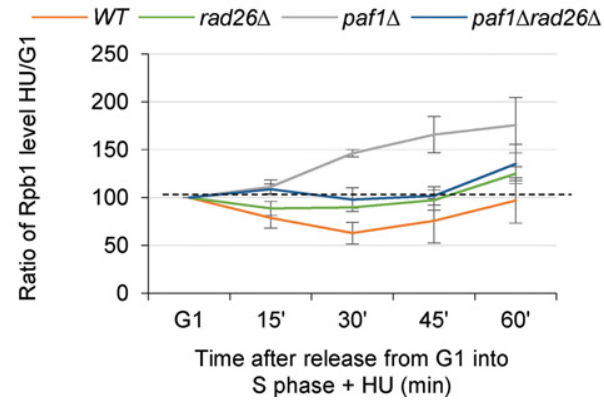

C

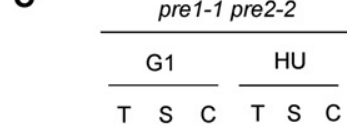

Rpb1

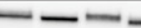

$\mathrm{Mcm} 2$

Tubulin
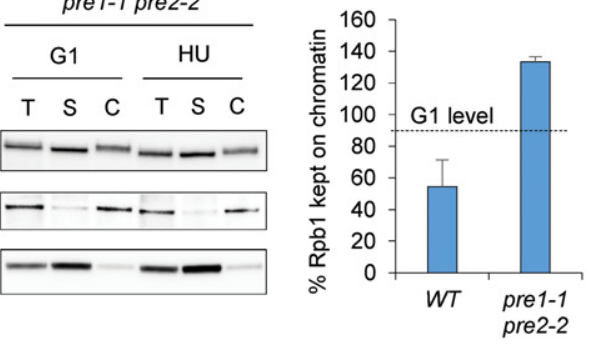

E

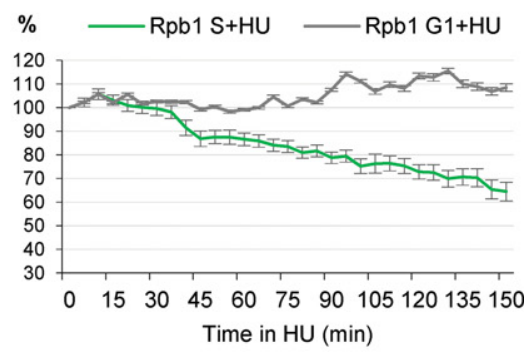

F

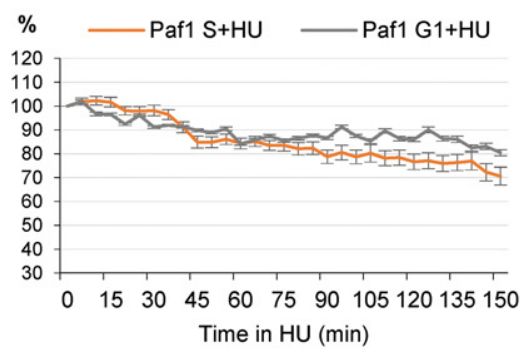

H

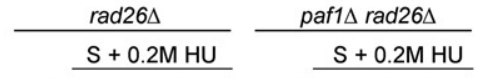

$\mathrm{Rpb} 1$

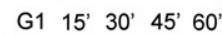

G1 15'30'45'60'

Tubulin
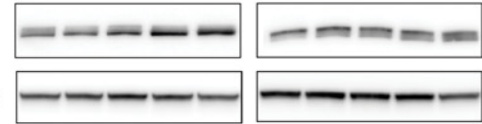

Figure 6. HU-induced degradation of RNAPII requires a functional DRC, INO80C, and PAF1C and does not involve TC-NER. Exponentially growing cells were synchronized in $\mathrm{G} 1$ with a factor and released in S phase in the presence of $0.2 \mathrm{M} H U$. $(A-C, G, H)$ Protein extracts were collected at the indicated times (in minutes) as either total protein $(A, B, G, H)$ or cellular fractions $(C)$. (T) Total proteins; (S) soluble proteins; (C) chromatin-associated proteins. Proteins were subjected to SDS-PAGE followed by immunoblotting with the indicated antibodies. Tub1 and $\mathrm{Mcm} 2$ were used as a loading control for total protein and chromatin fraction, respectively. (B,G) Quantitation of total $\mathrm{Rpb} 1$ after HU treatment, expressed as a percentage over the starting level in G1 (black line). Error bars represent SD of at least two individual experiments for each strain. (C) Quantitation of Rpb1 retained on chromatin after HU treatment. (D-F). Live single-cell analysis of Rpb1EGFP and Paf1-Ruby2 intensities in either G1 + HU or S phase + HU. (D) Representative images of yeast cells at the indicated time. (Green) Rpb1-EGFP; (red) Paf1-Ruby2. Bar, $2 \mu \mathrm{m}$. (E,F) Quantitation of Rpb1 and Paf1 fluorescence intensities, as indicated, measured on the same set of cells expressed as percentage over the G1 level (TO). Error bars are expressed as SEM (62 and 24 measurements on individual cells, respectively, for S + HU and G1 + HU). $(G, H)$ Quantitation of total Rpb1 levels in paf1 $\Delta$, rad26 $\Delta$, and paf1 1 rad26 upon release in S phase $+0.2 \mathrm{MHU}$ 


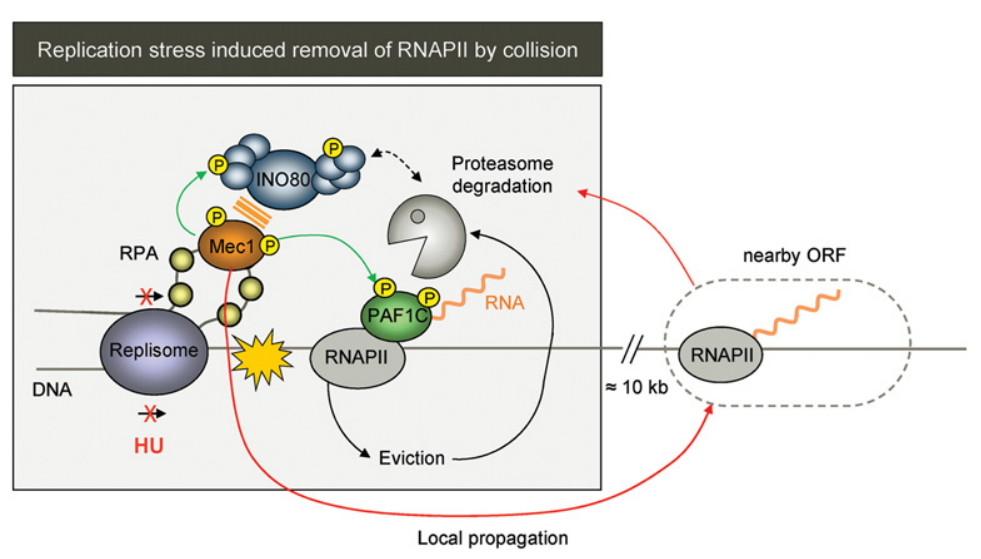

Figure 7. Schematic description of replication stress-induced removal and degradation of the transcriptional machinery at stalled forks mediated through Mec1-Ddc2, INO80C, and PAF1C. Upon HU-induced replication stress, Mec1-Ddc2 is activated by ssDNA. The kinase may modify RNAPII cofactor PAF1C and/or INO80C, both of which will be present on promoters of transcribing genes. Proximity to the fork-associated checkpoint kinase may activate the eviction of RNAPII-Ser5P and PAF1C, facilitated by INO80C at some promoters. RNAPII is then degraded by the proteasome near the site of collision or after eviction from chromatin. The system appears to preferentially act at genes transcribed in the same direction as the fork, and Mecl effects may propagate slightly ahead of the fork. the other mutations. On the other hand, as expected, there was a partial rescue of mec1 $1 \Delta \operatorname{sml} 1 \Delta$ sensitivity to UV exposure by rad26 (Supplemental Fig. S7D). Given that neither the UV nor the HU sensitivity of paf1 $1 \Delta$ or arp8s strains was alleviated by combining the mutation with rad264 (Supplemental Fig. S7D), we conclude that RNAPII degradation on $\mathrm{HU}$ is most likely distinct from the Rad26-Def1 pathway on UV (Fig. 6G,H), although there may be cross-talk on some level.

\section{Discussion}

Recent studies highlight the importance of coordinating transcription and replication machineries in S-phase cells. Multiple pathways ranging from RNA processing and transcriptional termination to specific helicases that remove DNA-RNA hybrids (Brambati et al. 2015) facilitate replication fork progression and promote genome stability. A recent report identified RNAPII itself as a dual-function factor that can block the progression of replication forks and favor replication restart, albeit by an unknown mechanism (Felipe-Abrio et al. 2015). Interestingly, Mec1-Ddc2 (ATR-ATRIP) was reported to help release actively transcribed genes from the nuclear periphery in order to limit topological impediments that might stall replication forks (Bermejo et al. 2011). On the other hand, no mechanism has been described to date for removal of the transcription machinery from DNA that is being replicated.

Here, we show that RNAPII is quickly evicted and degraded in response to $\mathrm{HU}$-induced replication stress in a manner that requires the RNAPII-associated complex PAF1 and the activity of Mec1 kinase (Fig. 7). This seems not to reflect a global shutdown of transcription but rather a local event that is controlled by activation of fork-associated Mec1-Ddc2. RNAPII eviction is also dependent on INO80C; the loss of INO80C integrity is synthetic-lethal on HU with mec1 or paf1 mutations (Fig. 1). INO80C has been shown to travel with the replication fork and facilitate replication fork restart after stalling (Papamichos-Chronakis and Peterson 2008; Shimada et al. 2008), yet it also maps to +1 nucleosomes at the transcription start site of many genes (Yen et al. 2013). Here we found INO80C implicated in eviction of RNAPII upon replica- tion stress. It acts additively with PAF1C and Mec1 kinase to promote cell survival on HU (Fig. 7). One way to achieve this cooperativity is by phosphorylating subunits of PAF1C and INO80C as part of the DRC. We note that although some subunits are targeted by other kinases, Mec1-Ddc2 does phosphorylate subunits of both complexes, and all three complexes influence the RNAPII eviction that we monitored on HU. The role of this mechanism may be to clear away RNAPII to allow replication fork restart after fork-RNAPII collision.

\section{Sites of replication fork-transcription collision show RNAPII eviction}

Recently, an elegant study demonstrated that most DSBs in HU-stressed wild-type cells map to active replication origins that fire near highly transcribed genes (Hoffman et al. 2015). Consistent with our observations, mec1 mutants exhibited an increased number of DSBs in the presence of HU compared with wild-type cells, probably reflecting the activation of late firing origins and the subsequent increase of replication-transcription conflicts. Among the high-frequency DSBs generated in the absence of Mec1 were breaks mapped in close proximity to the loci ARS106-PYK1 and ARS1219-YEF3, the two loci at which we scored Mec1-dependent removal of RNAPII. Thus, one role of the DRC may be to prevent replication fork collapse or DSBs by promoting the removal of RNAPII where transcription and replication forks are likely to collide. The loci at which we monitored this phenomenon are very close to origins of replication and either have been replicated or will soon be by $2 \mathrm{~h}$ on HU (Supplemental Fig. S4). This mechanism could account for the loss of viability observed in strains that are mutated for individual components of these complexes and the additivity observed when such mutants are combined (Fig. 1). The pathway is summarized in Figure 7.

The degradation of RNAPII that we detected on HU is reminiscent of the UV damage-induced degradation of stalled RNAPII (Gaillard and Aguilera 2013). In this latter phenomenon, Def1 promotes RNAPII degradation by ubiquitination, whereas the TC-NER factor Rad26/CSB inhibits this degradation (Gaillard and Aguilera 2013). 
Interestingly, in the absence of both Rad26 and Def1, RNAPII degradation regains wild-type kinetics, suggesting the involvement of other pathways that ensure survival (Woudstra et al. 2002). Although it has been reported that Mec1 promotes TC-NER activation after UV treatment by phosphorylating Rad26 (Taschner et al. 2010), $\operatorname{rad} 26 \Delta$ neither enhances RNAPII degradation nor increases sensitivity to HU by drop assays (Supplemental Fig. S7D). Furthermore, we did not see an increase, but rather a reproducible drop, in the efficiency of RNAPII degradation on HU in rad26 cells on HU when compared with wildtype strains. Consistently, there was no rescue observed for double mutants in which rad26 $\Delta$ is combined with either the mec1 1 , paf1 $\Delta$, or arp $8 \Delta$ mutation.

A further argument for the independence of these pathways is that RAD26 ablation in a paf1 $\triangle$ mutant does not rescue the RNAPII degradation defect on HU. Thus, the TC-NER pathway appears to be distinct from the eviction of RNAPII machinery in the context of replication fork stalling. Moreover, the eviction provoked by transcription-replication fork interference is an immediate response, with RNAPII being degraded rapidly (15 min after release into $\mathrm{HU}$, with a peak at $30 \mathrm{~min}$ ), while $\mathrm{UV}$-induced degradation is much slower (Harreman et al. 2009). We did see an effect of rad26 deletion on a paf1 $\Delta$ strain treated with HU, in that the accumulation of RNAPII that occurs after long periods of incubation is reduced (Fig. 6; Supplemental Fig. S7A).

Although transcription-replication interference may also occur during progression through a normal S phase, there was no global reduction of chromatin-bound Rpb1 in an unperturbed population of S-phase cells. This is consistent with the fact that Mec1 modifies largely different sets of proteins in unperturbed S-phase cells than it does in S-phase cells under stress (Hoch et al. 2013; Bastos de Oliveira et al. 2015). Whereas HU-induced removal of RNAPII from chromatin requires $\mathrm{Mec1}$, it does not require the downstream kinase Rad53/CHK2. This is reinforced by our observation that some phosphoacceptor sites in INO80C and PAF1C subunits on HU are dependent on Mec1 but not Rad53 (Hustedt et al. 2015). Indeed, both Mec1 and Rad53 make distinct contributions toward cell survival in the face of replicative stress (Hustedt et al. 2013). It remains to be seen whether modification by checkpoint kinases alters the activity and/or function of INO80C and PAF1C in this context.

\section{A novel role for PAF1C in polymerase eviction}

Our results indicate that PAF1C inactivation leads to defects in replication fork progression and restart on $\mathrm{HU}$ that are similar to mutations in INO80C or Mec1 (Fig. 4). However, unlike INO80C, Mec1, and the termination factor Sen1, which bind replication forks on HU (Shimada et al. 2008; Alzu et al. 2012), there is no enrichment of PAF1C at early or late origins. This suggests that PAF1C is present at sites of transcription-replication interference due to its interaction with the transcribing RNAPII. Mec1-induced dissociation of PAF1C from the RNAPII complex might help to remodel/destabilize the transcrip- tion machinery, favoring Rbp1 removal from chromatin and/or its degradation.

The requirement for INO80C to cope with HU stress was associated with a defect in PCNA ubiquitination by Rad18 and impaired Rad51-mediated restart of replication (Falbo et al. 2009). On the other hand, INO80C is found at ARS elements and may play a role in normal fork elongation through chromatin (Shimada et al. 2008). Our data suggest that INO80C contributes to RNAPII eviction and degradation following $\mathrm{HU}$ treatment and extend a study that links INO80C to Cdc48 and the proteasome for the removal of RNAPII from chromatin in the presence of MMS (Lafon et al. 2015). Interestingly, DNA damageindependent degradation of RNAPII seems to occur on chromatin, since Cdc48 and several subunits of the proteasome are recruited to transcribed genes (Verma et al. 2011; Karakasili et al. 2015). Our data argue that RNAPII eviction and degradation that we observed on HU are coupled, since we could not observe a reduction of chromatinassociated RNAPII in a pre 1-1 pre2-2 proteasome mutant. Still, it remains to be addressed whether RNAPII is first evicted and then degraded or whether RNAPII degradation happens directly at chromatin sites of fork stalling.

Much like FACT, a chromatin remodeler that also reduces R-loop formation to enable replication fork progression through transcribed regions (Herrera-Moyano et al. 2014), INO80C was reported to limit the abundance of spurious and unstable noncoding RNA transcripts (Alcid and Tsukiyama 2014). This may be related to the RNAPII eviction that we scored on HU. In cells lacking INO80C, R-loop accumulation is expected to increase, which would aggravate replication problems incurred by the presence of HU. Although the number of specific loci studied here is small, Mec1-dependent removal of RNAPII was not observed at two loci that are predicted to generate head-on collisions, in contrast to Sen1, which appears to act only at such sites (Alzu et al. 2012). Rather, Mec1 effects were observed at loci transcribed codirectionally with the nearest replication fork. It has been observed that head-on collisions are more detrimental to cell survival (Prado and Aguilera 2005). Thus, if Mec1 proves to remove only codirectional RNAPII at the genome-wide level, its ability to clean up these, but not head-on collisions, might explain why these latter generate more genomic instability in wild-type cells.

\section{Locus-specific RNAPII eviction on $\mathrm{HU}$}

Variability in the response to transcription-replication interference may also be due to the set of transcription factors and specific features of the chromatin found at the gene of interest. At the four loci tested by ChIP-qPCR, RNAPII removal was not impaired in the INO80C mutant $\operatorname{arp} 8 \Delta$ (Fig. 5; Supplemental Fig. S5), yet this may mean that INO80C was not originally bound at these loci. Our data do suggest that RNAPII removal is, to some extent, locus-specific, although multiple pathways exist to resolve transcription-replication interference. The fact that Mec1, PAF1C, and INO80C act at least partially on nonoverlapping genomic loci is consistent with their 
synergistic effects on viability in double or triple mutants on HU.

We detected for the first time interactions between Mec1 and INO80C and between INO80C and PAF1C and present evidence for larger complexes being formed (Fig. 2). Most likely, Mec1-Dde2 is brought to sites of collision by the replication machinery, while PAF1C accumulates there due to transcribing RNAPII. INO80C recruitment may be influenced in part by promoter-specific factors. We note that Mecl also forms a transient complex with the SWI/SNF complex, which is another chromatin remodeler implicated in both DSB repair and transcriptional control (Kapoor et al. 2015). Transient interactions such as these may be mediated by post-translational modifications, allowing rapid translation of signal cascades into chromatin configurations. Future studies will be directed toward elucidating the exact contribution made by each complex and the impact of Mec1-Ddc2 on their respective activities. It will also be important to determine the conservation of this pathway, which helps suppress the genome instability that arises from collisions between transcription machinery/R loops and replication forks.

\section{Materials and methods}

Yeast strains, cultures, and flow cytometry

All strains used are listed in Supplemental Table S1. YEP medium was supplemented with $2 \%$ glucose. MATa cells were synchronized in $\mathrm{G}_{1}$ by adding $5 \mu \mathrm{g} \mathrm{mL^{-1 }}$ a factor for $170 \mathrm{~min}$ at $25^{\circ} \mathrm{C}$. $\mathrm{G}_{1}$-blocked cells were released into $\mathrm{S}$ phase $\pm 0.2 \mathrm{M} \mathrm{HU}$. Flow cytometry samples were prepared as previously described (Haase and Lew 1997). Data were acquired on a FACScalibur (Becton Dickinson) and analyzed with Cell Quest software.

\section{Fractionation, protein extracts, and Western blotting}

Fractionation was performed as previously described (Pasero et al. 1999|. TCA precipitated protein extracts were resolved by SDSPAGE (criterion: TGX 4\%-15\%; Bio-Rad). Western blotting was performed with a mouse monoclonal anti-Rpb1-CTD (8WG16; Abcam, ab817), a mouse monoclonal anti-tubulin (Woods et al. 1989), and a goat polyclonal ant-Mcm2 (yN-19; Santa Cruz Biotechnology, sc-6680). Total protein extracts were normalized for tubulin, whereas chromatin fraction were normalized to $\mathrm{Mcm} 2$. Blots were scanned with an ImageQuant LAS4000 Mini (GE healthcare), and semiquantitative determination of protein level was performed using ImageJ (Fiji) software.

\section{RNAPII and Paf1 ChIP}

ChIP-qPCR was performed as described in Katou et al. (2003) using anti-PK clone SV5-Pk1 (Serotec) and anti-Rpb1-CTD (8WG16; Abcam, ab817) coupled to Dynabeads (Invitrogen; protein A and sheep anti-mouse M280 IgG). For quantitative PCR, background controls were determined using uncoupled Dynabeads, and enrichment was normalized to chromatin input and transcriptionnegative site. Primers are available on request.

\section{DNA combing}

DNA combing was performed as described (Bianco et al. 2012) using a mouse monoclonal anti-ssDNA (Chemicon, clone 16-19) and a rat monoclonal anti-BrdU (Abcys, clone BU1/75). Images were recorded on a Leica DM6000 microscope equipped with a CoolSNAP HQ CCD camera (Roper Scientific) and were processed as described (Bianco et al. 2012).

\section{EMAP}

The EMAP analysis was performed as described in Guenole et al. (2013), and suppressive (not synergistic) effects were elucidated in Hustedt et al. (2015).

Endogenous purification of INO80-Mec1-PAF1 and XL-MS

A $6 x$ His tag was introduced after the coding region of MEC1 in the background of a double-Flag-tagged INO80 (Shen et al. 2000), and the purification and cross-link studies are described in the Supplemental Material.

\section{Coimmunoprecipitation}

Yeast strains were harvested in log phase, washed in HN0.5 (25 mM HEPES at pH 7.9, $500 \mathrm{mM} \mathrm{NaCl}, 10 \%$ [v/v] glycerol, $0.05 \%[\mathrm{v} / \mathrm{v}] \mathrm{NP} 40,1 \mathrm{mM}$ EDTA, $1 \mathrm{mM}$ DTT), resuspended in HN0.5 containing Complete protease inhibitor mix (Roche), and disrupted with zirconia/silica beads (BioSpec) in a MP FastPrep24. Immunoprecipitation was first performed using antiFlag M2 magnetic beads (Sigma, M8823). Beads were optionally washed with at least $10 \times$ bead volume benzonase buffer $(25 \mathrm{mM}$ HEPES at pH 7.9, $100 \mathrm{mM} \mathrm{NaCl}, 10 \%$ [v/v] glycerol, $0.05 \%$ [v/ v] NP40, $2 \mathrm{mM} \mathrm{MgCl}_{2}, 1 \mathrm{mM}$ DTT) and then treated for $30 \mathrm{~min}$ with $>1000 \mathrm{U}$ of benzonase nuclease (Sigma). Beads were then washed with at least $25 \times$ bead volume HNO.5 and then with $12 \times$ bead volume HN0.1 (25 mM HEPES at $\mathrm{pH} 7.9,100 \mathrm{mM}$ $\mathrm{NaCl}, 10 \%[\mathrm{v} / \mathrm{v}$ ] glycerol, $0.05 \%$ [v/v] NP40, 1 mM EDTA, $1 \mathrm{mM}$ DTT). Ino80-Flag elution was performed with 4x bead volumes of $200 \mathrm{ng} / \mathrm{\mu L} 3 x$ Flag peptide (Sigma) in HNO.1. Flag elution was incubated with either Dynabeads Prot G (Life Technologies) coupled to anti-PK (V5) antibody (SM 1691, Acris) or uncoupled beads as a control. Beads were washed with at least $30 \times$ bead volume HNO.1, and bound proteins were subjected to SDS-PAGE and Western blotting with the same anti-Flag M2, anti-PK, or anti-HA F7 (SC7392). Alternatively, immunoprecipitation was performed with beads coupled to purified anti-Arp5 antibody (Shimada et al. 2008) on extracts from cells lacking the Flag tag.

\section{Purification of soluble PAF1C}

Overnight culture of yeast strain GA-9524 with a TEV proteasecleavable $9 \times$ PK tag was harvested, washed in HNO.5, resuspended in HNO.5 containing Complete protease inhibitor mix (Roche), and disrupted with zirconia/silica beads (BioSpec) in a MP Fast-Prep24. Cell extract was incubated with anti-PK (V5) antibody (SM 1691; Acris) coupled to Dynabeads Prot G (Life Technologies). Beads were washed with at least $30 \times$ bead volume HN0.5, and PAF1C was solubilized by overnight cleavage with $3 \mu \mathrm{g}$ of TEV protease. TEV protease was removed via concentration of PAF1C in an Amicon centrifugal filter with $100 \mathrm{kDa}$ of MWCO.

Kinase assays and mass spectroscopic analysis of phosphopeptides

The radioactive $\gamma^{3}{ }^{32}$ PATP kinase assay was performed as described (Hustedt et al. 2015). Myc-tagged Mec1 was recovered 
by immunoprecipitation and titrated into a fixed amount of acceptor peptide PHAS- 1 or incubated with purified PAF1C with or without $30 \mathrm{mM}$ caffeine for $30 \mathrm{~min}$ at $30^{\circ} \mathrm{C}$. The Flag-purified INO80C-Mec1-Ddc2 complex was added at $\sim 90,45$, and $12.5 \mathrm{ng}$ to the PHAS-1 substrate with $\gamma-{ }^{32} \mathrm{P}$-ATP. ${ }^{32} \mathrm{P}$ incorporation was monitored by phosphorimaging. Identification of phosphopeptides was done as described previously (Hustedt et al. 2015).

\section{Microscopy}

Live microscopy used an Olympus IX81 microscope equipped with a Yokogawa CSU-X1 scan head, an EM-CCD Cascade II (Photometrics), a ASI MS-2000 Z-piezo stage, and a PlanApo $100 \times, 1.45 \mathrm{NA}$ total internal reflection fluorescence microscope oil objective. Fluorophores were excited at $561 \mathrm{~nm}$ (mCherry, $\sim 30 \mu \mathrm{W})$ and 491 (GFP, $\sim 75 \mu \mathrm{W})$. Bright-field images were acquired using a CoolLED diode. Imaging was performed in filtered, nonautoclaved YPAD using an Onix CellAsic microfluidic chamber to regulate cell synchronization in a factor and the release into $200 \mathrm{mM}$ HU. Time-lapse series (150 min total) of 50 optical slices per stack were acquired for 30 time points at intervals of $5 \mathrm{~min}$, with each slice being exposed for $30 \mathrm{msec}$ per laser line. Images were deconvolved using Huygens Pro and channel-aligned. Lasers were used for the 561 and 491 lines at $6 \%$ and $5 \%$, respectively. Bleaching did not occur in these conditions until $5 \mathrm{~h}$.

\section{Image analysis}

Deconvolved images were analyzed as a merged stack in ICY. Nuclei were detected and segmented using HK means and active contours and followed through the time series. The integrated nuclear intensity was calculated for each cell nucleus.

\section{Acknowledgments}

We thank E. Schwob and the DNA combing facility of Montpellier for silanized coverslips, and the Montpellier RIO Imaging microscopy facility. We thank M. Papamichos-Chronakis for sharing information before publication. J.P. is supported by a fellowship from the French association Fondation pour la Recherche sur le Cancer (ARC) and an EMBO long-term fellowship (ALTF736-2015). C.-B.G. holds a FP7 Marie Curie Intra European Fellowship (626708). S.M.G. gratefully acknowledges support of the Novartis Research Foundation, the Swiss National Science Foundation, and a Human Frontier Science Program grant. K.P. $\mathrm{H}$ acknowledges support by the European Research Council (Advanced Grant ATMMACHINE), the German Research Council (SFB1064), and the Center for Integrated Protein Science of the German Excellence Initiative. J.P., C.-B.G., N.H., A.T., K.S., P.P., K.-P.H., and S.M.G. designed the experiments. J.P., C.-B.G., N.H., K.S., and A.T. performed the experiments. F.H. analyzed the cross-link data. R.S. performed enrichment and measurements of the phosphopeptides. J.P., C.-B.G., and S.M.G. wrote the manuscript.

\section{References}

Alcid EA, Tsukiyama T. 2014. ATP-dependent chromatin remodeling shapes the long noncoding RNA landscape. Genes DeV 28: $2348-2360$.

Alzu A, Bermejo R, Begnis M, Lucca C, Piccini D, Carotenuto W, Saponaro M, Brambati A, Cocito A, Foiani M, et al. 2012. Senataxin associates with replication forks to protect fork integri- ty across RNA-polymerase-II-transcribed genes. Cell 151: 835-846.

Azvolinsky A, Giresi PG, Lieb JD, Zakian VA. 2009. Highly transcribed RNA polymerase II genes are impediments to replication fork progression in Saccharomyces cerevisiae. Mol Cell 34: 722-734.

Bastos de Oliveira FM, Kim D, Cussiol JR, Das J, Jeong MC, Doerfler L, Schmidt KH, Yu H, Smolka MB. 2015. Phosphoproteomics reveals distinct modes of Mec1/ATR signaling during DNA replication. Mol Cell 57: 1124-1132.

Bermejo R, Capra T, Jossen R, Colosio A, Frattini C, Carotenuto W, Cocito A, Doksani Y, Klein H, Gomez-Gonzalez B, et al. 2011. The replication checkpoint protects fork stability by releasing transcribed genes from nuclear pores. Cell 146: 233-246.

Bianco JN, Poli J, Saksouk J, Bacal J, Silva MJ, Yoshida K, Lin YL, Tourriere H, Lengronne A, Pasero P. 2012. Analysis of DNA replication profiles in budding yeast and mammalian cells using DNA combing. Methods (San Diego, Calif) 57: 149-157.

Brambati A, Colosio A, Zardoni L, Galanti L, Liberi G. 2015. Replication and transcription on a collision course: eukaryotic regulation mechanisms and implications for DNA stability. Front Genet 6: 166.

Branzei D, Foiani M. 2010. Maintaining genome stability at the replication fork. Nat Rev Mol Cell Biol 11: 208-219.

Chang M, French-Cornay D, Fan HY, Klein H, Denis CL, Jaehning JA. 1999. A complex containing RNA polymerase II, Paflp, Cdc73p, Hpr1p, and Ccr4p plays a role in protein kinase C signaling. Mol Cell Biol 19: 1056-1067.

Chen S-H, Albuquerque CP, Liang J, Suhandynata RT, Zhou H. 2010. A proteome-wide analysis of kinase-substrate network in the DNA damage response. J Biol Chem 285: 12803-12812.

Chen L, Conaway RC, Conaway JW. 2013. Multiple modes of regulation of the human Ino80 SNF2 ATPase by subunits of the INO80 chromatin-remodeling complex. Proc Natl Acad Sci 110: 20497-20502.

Cobb JA, Schleker T, Rojas V, Bjergbaek L, Tercero JA, Gasser SM. 2005. Replisome instability, fork collapse, and gross chromosomal rearrangements arise synergistically from Mec1 kinase and RecQ helicase mutations. Genes Dev 19: 3055-3069.

Collins SR, Miller KM, Maas NL, Roguev A, Fillingham J, Chu CS, Schuldiner M, Gebbia M, Recht J, Shales M, et al. 2007. Functional dissection of protein complexes involved in yeast chromosome biology using a genetic interaction map. Nature 446: 806-810.

Dronamraju R, Strahl BD. 2014. A feed forward circuit comprising Spt6, Ctk1 and PAF regulates Pol II CTD phosphorylation and transcription elongation. Nucleic Acids Res 42: 870-881.

Duch A, Felipe-Abrio I, Barroso S, Yaakov G, Garcia-Rubio M, Aguilera A, de Nadal E, Posas F. 2013. Coordinated control of replication and transcription by a SAPK protects genomic integrity. Nature 493: 116-119.

El Hage A, Webb S, Kerr A, Tollervey D. 2014. Genome-wide distribution of RNA-DNA hybrids identifies RNase $\mathrm{H}$ targets in tRNA genes, retrotransposons and mitochondria. PLoS Genet 10: e1004716.

Falbo KB, Alabert C, Katou Y, Wu S, Han J, Wehr T, Xiao J, He X, Zhang Z, Shi Y, et al. 2009. Involvement of a chromatin remodeling complex in damage tolerance during DNA replication. Nat Struct Mol Biol 16: 1167-1172.

Felipe-Abrio I, Lafuente-Barquero J, Garcia-Rubio ML, Aguilera A. 2015. RNA polymerase II contributes to preventing transcription-mediated replication fork stalls. $E M B O J \mathbf{3 4}$ 236-250. 
Gaillard H, Aguilera A. 2013. Transcription coupled repair at the interface between transcription elongation and mRNP biogenesis. Biochim Biophys Acta 1829: 141-150.

Gerhold CB, Hauer MH, Gasser SM. 2015. INO80-C and SWR-C: guardians of the genome. J Mol Biol 427: 637-651.

Gomez-Gonzalez B, Garcia-Rubio M, Bermejo R, Gaillard H, Shirahige K, Marin A, Foiani M, Aguilera A. 2011. Genomewide function of THO/TREX in active genes prevents Rloop-dependent replication obstacles. EMBO J 30: 3106-3119.

Gonzalez-Aguilera C, Tous C, Gomez-Gonzalez B, Huertas P, Luna R, Aguilera A. 2008. The THP1-SAC3-SUS1-CDC31 complex works in transcription elongation-mRNA export preventing RNA-mediated genome instability. Mol Biol Cell 19: 4310-4318.

Guenole A, Srivas R, Vreeken K, Wang ZZ, Wang S, Krogan NJ, Ideker T, van Attikum H. 2013. Dissection of DNA damage responses using multiconditional genetic interaction maps. Mol Cell 49: 346-358.

Haase SB, Lew DJ. 1997. Flow cytometric analysis of DNA content in budding yeast. Methods Enzymol 283: 322-332.

Harreman M, Taschner M, Sigurdsson S, Anindya R, Reid J, Somesh B, Kong SE, Banks CA, Conaway RC, Conaway JW, et al. 2009. Distinct ubiquitin ligases act sequentially for RNA polymerase II polyubiquitylation. Proc Natl Acad Sci 106: 20705-20710.

Herrera-Moyano E, Mergui X, Garcia-Rubio ML, Barroso S, Aguilera A. 2014. The yeast and human FACT chromatin-reorganizing complexes solve R-loop-mediated transcriptionreplication conflicts. Genes Dev 28: 735-748.

Hoch NC, Chen ES, Buckland R, Wang SC, Fazio A, Hammet A, Pellicioli A, Chabes A, Tsai MD, Heierhorst J. 2013. Molecular basis of the essential s phase function of the rad53 checkpoint kinase. Mol Cell Biol 33: 3202-3213.

Hoffman EA, McCulley A, Haarer B, Arnak R, Feng W. 2015. Break-seq reveals hydroxyurea-induced chromosome fragility as a result of unscheduled conflict between DNA replication and transcription. Genome Res 25: 402-412.

Hustedt N, Gasser SM, Shimada K. 2013. Replication checkpoint: tuning and coordination of replication forks in s phase. Genes 4: $388-434$.

Hustedt N, Seeber A, Sack R, Tsai-Pflugfelder M, Bhullar B, Vlaming $\mathrm{H}$, van Leeuwen F, Guénolé A, van Attikum H, Srivas R, et al. 2015. Yeast PP4 interacts with ATR homolog Ddc2Mecl and regulates checkpoint signaling. Mol Cell 57: 273-289.

Jaehning JA. 2010. The Paf1 complex: platform or player in RNA polymerase II transcription? Biochim Biophys Acta 1799: 379-388.

Kapoor P, Bao Y, Xiao J, Luo J, Shen J, Persinger J, Peng G, Ranish J, Bartholomew B, Shen X. 2015. Regulation of Mec1 kinase activity by the SWI/SNF chromatin remodelling complex. Genes Dev 29: 591-602.

Karakasili E, Burkert-Kautzsch C, Kieser A, Strasser K. 2015. Degradation of DNA damage-independently stalled RNA polymerase II is independent of the E3 ligase Elc1. Nucleic Acids Res 43: 2486.

Katou Y, Kanoh Y, Bando M, Noguchi H, Tanaka H, Ashikari T, Sugimoto K, Shirahige K. 2003. S-phase checkpoint proteins Tof 1 and Mrc 1 form a stable replication-pausing complex. $\mathrm{Na}$ ture 424: 1078-1083.

Lafon A, Taranum S, Pietrocola F, Dingli F, Loew D, Brahma S, Bartholomew B, Papamichos-Chronakis M. 2015. INO80 chromatin remodeler facilitates release of RNA polymerase II from chromatin for ubiquitin-mediated proteasomal degradation. Mol Cell 60: 784-796.
Leitner A, Walzthoeni T, Kahraman A, Herzog F, Rinner O, Beck M, Aebersold R. 2010. Probing native protein structures by chemical cross-linking, mass spectrometry, and bioinformatics. Mol Cell Proteomics 9: 1634-1649.

Mallory JC, Petes TD. 2000. Protein kinase activity of Tellp and Meclp, two Saccharomyces cerevisiae proteins related to the human ATM protein kinase. Proc Natl Acad Sci 97: 13749-13754.

Mayer A, Lidschreiber M, Siebert M, Leike K, Soding J, Cramer P. 2010. Uniform transitions of the general RNA polymerase II transcription complex. Nat Struct Mol Biol 17: 1272-1278.

Morrison AJ, Kim J-A, Person MD, Highland J, Xiao J, Wehr TS, Hensley S, Bao Y, Shen J, Collins SR, et al. 2007. Mec1/Tel1 phosphorylation of the INO80 chromatin remodeling complex influences DNA damage checkpoint responses. Cell 130: 499-511.

Nguyen VC, Clelland BW, Hockman DJ, Kujat-Choy SL, Mewhort HE, Schultz MC. 2010. Replication stress checkpoint signaling controls tRNA gene transcription. Nat Struct Mol Biol 17: 976-981.

Paciotti V, Clerici M, Lucchini G, Longhese MP. 2000. The checkpoint protein Ddc2, functionally related to $S$. pombe Rad26, interacts with Mec1 and is regulated by Mec1-dependent phosphorylation in budding yeast. Genes Dev 14: 2046-2059.

Papamichos-Chronakis M, Peterson CL. 2008. The Ino80 chromatin-remodeling enzyme regulates replisome function and stability. Nat Struct Mol Biol 15: 338-345.

Pasero P, Duncker BP, Schwob E, Gasser SM. 1999. A role for the $\mathrm{Cdc} 7$ kinase regulatory subunit Dbf4p in the formation of initiation-competent origins of replication. Genes Dev 13: 2159-2176.

Penheiter KL, Washburn TM, Porter SE, Hoffman MG, Jaehning JA. 2005. A posttranscriptional role for the yeast Paf1-RNA polymerase II complex is revealed by identification of primary targets. Mol Cell 20: 213-223.

Poli J, Tsaponina O, Crabbe L, Keszthelyi A, Pantesco V, Chabes A, Lengronne A, Pasero P. 2012. dNTP pools determine fork progression and origin usage under replication stress. EMBO J 31: 883-894.

Prado F, Aguilera A. 2005. Impairment of replication fork progression mediates RNA polII transcription-associated recombination. EMBO J 24: 1267-1276.

Santos-Pereira JM, Aguilera A. 2015. R loops: new modulators of genome dynamics and function. Nat Rev Genet 16: 583-597.

Shen X. 2004. Preparation and analysis of the INO80 complex. Methods Enzymol 377: 401-412.

Shen X, Mizuguchi G, Hamiche A, Wu C. 2000. A chromatin remodelling complex involved in transcription and DNA processing. Nature 406: 541-544.

Shimada K, Oma Y, Schleker T, Kugou K, Ohta K, Harata M, Gasser SM. 2008. Ino80 chromatin remodeling complex promotes recovery of stalled replication forks. Curr Biology 18: 566-575.

Somesh BP, Reid J, Liu WF, Sogaard TM, Erdjument-Bromage H, Tempst P, Svejstrup JQ. 2005. Multiple mechanisms confining RNA polymerase II ubiquitylation to polymerases undergoing transcriptional arrest. Cell 121: 913-923.

Taschner M, Harreman M, Teng Y, Gill H, Anindya R, Maslen SL, Skehel JM, Waters R, Svejstrup JQ. 2010. A role for checkpoint kinase-dependent Rad26 phosphorylation in transcriptioncoupled DNA repair in Saccharomyces cerevisiae. Mol Cell Biol 30: 436-446.

Tosi A, Haas C, Herzog F, Gilmozzi A, Berninghausen O, Ungewickell C, Gerhold CB, Lakomek K, Aebersold R, Beckmann $\mathrm{R}$, et al. 2013. Structure and subunit topology of the INO80 
Poli et al.

chromatin remodeler and its nucleosome complex. Cell 154: 1207-1219.

Tourriere H, Pasero P. 2007. Maintenance of fork integrity at damaged DNA and natural pause sites. DNA Repair 6: 900-913.

Tuduri S, Crabbe L, Conti C, Tourriere H, Holtgreve-Grez H, Jauch A, Pantesco V, De Vos J, Thomas A, Theillet C, et al. 2009. Topoisomerase I suppresses genomic instability by preventing interference between replication and transcription. Nat Cell Biol 11: 1315-1324.

Verma R, Oania R, Fang R, Smith GT, Deshaies RJ. 2011. Cdc48/ p97 mediates UV-dependent turnover of RNA Pol II. Mol Cell 41: 82-92.

Wade PA, Werel W, Fentzke RC, Thompson NE, Leykam JF, Burgess RR, Jaehning JA, Burton ZF. 1996. A novel collection of accessory factors associated with yeast RNA polymerase II. Protein Exp Purif 8: 85-90.

Wahba L, Amon JD, Koshland D, Vuica-Ross M. 2011. RNase H and multiple RNA biogenesis factors cooperate to prevent RNA:DNA hybrids from generating genome instability. Mol Cell 44: 978-988.

Watanabe S, Tan D, Lakshminarasimhan M, Washburn MP, Hong EJ, Walz T, Peterson CL. 2015. Structural analyses of the chromatin remodelling enzymes INO80-C and SWR-C. Nat Commun 6: 7108.
Wellinger RE, Prado F, Aguilera A. 2006. Replication fork progression is impaired by transcription in hyperrecombinant yeast cells lacking a functional THO complex. Mol Cell Biol 26: 3327-3334.

Wood A, Schneider J, Dover J, Johnston M, Shilatifard A. 2003. The Paf1 complex is essential for histone monoubiquitination by the Rad6-Bre1 complex, which signals for histone methylation by COMPASS and Dotlp. I Biol Chem 278: 34739-34742.

Woods A, Sherwin T, Sasse R, MacRae TH, Baines AJ, Gull K. 1989. Definition of individual components within the cytoskeleton of Trypanosoma brucei by a library of monoclonal antibodies. J Cell Sci 93(Pt 3): 491-500.

Woudstra EC, Gilbert C, Fellows J, Jansen L, Brouwer J, Erdjument-Bromage H, Tempst P, Svejstrup JQ. 2002. A Rad26Def1 complex coordinates repair and RNA Pol II proteolysis in response to DNA damage. Nature 415: 929-933.

Xue Y, Van C, Pradhan SK, Su T, Gehrke J, Kuryan BG, Kitada T, Vashisht A, Tran N, Wohlschlegel J, et al. 2015. The Ino80 complex prevents invasion of euchromatin into silent chromatin. Genes Dev 29: 350-355.

Yen K, Vinayachandran V, Pugh BF. 2013. SWR-C and INO80 chromatin remodcelers recognize nucleosome-free regions near +1 nucleosomes. Cell 154: 1246-1256.

Zeman MK, Cimprich KA. 2014. Causes and consequences of replication stress. Nat Cell Biol 16: 2-9. 


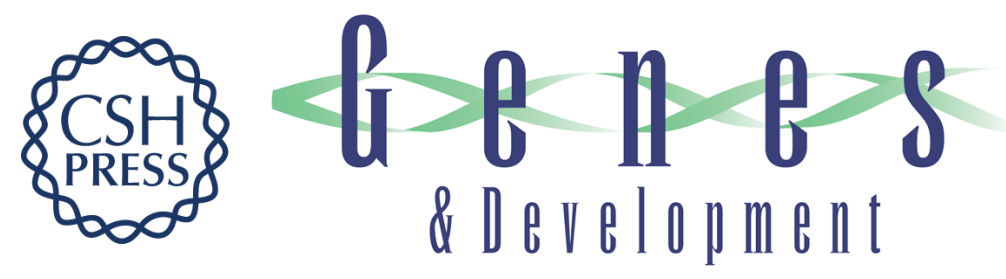

\title{
Mec1, INO80, and the PAF1 complex cooperate to limit transcription replication conflicts through RNAPII removal during replication stress
}

\author{
Jérôme Poli, Christian-Benedikt Gerhold, Alessandro Tosi, et al.
}

Genes Dev. 2016, 30: originally published online January 21, 2016

Access the most recent version at doi:10.1101/gad.273813.115

\section{Supplemental http://genesdev.cshlp.org/content/suppl/2016/01/20/gad.273813.115.DC1 Material}

References

Creative

Commons

License

Email Alerting
Service
This article cites 66 articles, 24 of which can be accessed free at: http://genesdev.cshlp.org/content/30/3/337.full.html\#ref-list-1

This article is distributed exclusively by Cold Spring Harbor Laboratory Press for the first six months after the full-issue publication date (see

http://genesdev.cshlp.org/site/misc/terms.xhtml). After six months, it is available under a Creative Commons License (Attribution-NonCommercial 4.0 International), as described at http://creativecommons.org/licenses/by-nc/4.0/.

Receive free email alerts when new articles cite this article - sign up in the box at the top right corner of the article or click here.

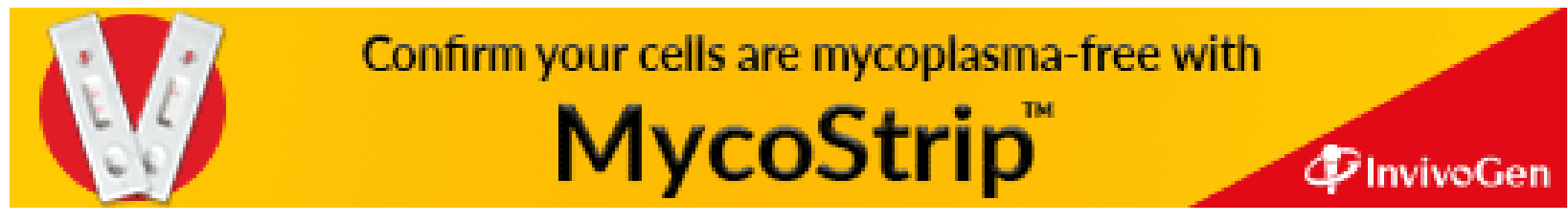

